\title{
PROBLEMÁTICA DE LOS FECHADOS CARBONO 14 Y SU CALIBRACIÓN: EL CASO DEL FORMATIVO DE LOS ANDES CENTRALES
}

\author{
Leonid Velarde
}

\begin{abstract}
Sumilla:
El siguiente artículo presenta las bases de la datación radiocarónica, los problemas que puede presentar una muestra y los métodos de calibración para corregir la desviación de los fechados radiocarbónicos. El autor, usando fechados del Período Formativo de los Andes Centrales, nos muestra diferentes ejemplos de muestras calibradas y su efecto en la cronología del periodo.
\end{abstract}

\section{Abstract:}

The following paper presents the basis of the radiocarbon dating, the possible problems in a sample to be dated and the methods of calibration used to correct the deviation of radiocarbon dates. Using differents radiocarbonic dates of the Central Andes Formative period, the author analyses calibrated dates and their effect over the period chronology.

\section{INTRODUCCIÓN}

La problemática del Período Formativo de los Andes Centrales se encuentra siempre en actualidad. Lamentablemente, existe la tendencia a discutir su duración temporal en términos "Calendárico-históricos" tomando en cuenta fechados "absolutos" obtenidos mediante el método llamado Carbono $14\left({ }^{14} \mathrm{C}\right)$. Las fechas así obtenidas conforman un calendario radiocarbónico el cual está sujeto a alteraciones debidas, principalmente, a una constante variación de la concentración de ${ }^{14} \mathrm{C}$ en la atmósfera. Para poder solucionar este problema se vienen desarrollando constantemente diferentes métodos llamados de "calibración" o impropiamente de "corrección", los cuales buscan dar una mayor coherencia en "tiempo real" a los fechados encontrados. A su vez, los métodos de datación ${ }^{14} \mathrm{C}$ se vuelven más precisos. Es en atención a estos nuevos datos, técnicas y métodos, que creemos necesario exponer la problemática de las fechas absolutas, tomando como ejemplo el Formativo peruano.

El presente trabajo fue realizado entre 1994-1995 como un primer intento de entendimiento de la problemática de la cronología del Formativo en el marco de la tesis de doctorado que venimos realizando en la Universidad de Ginebra. Debemos aclarar que para poder obtener la mayor cantidad de datos originales, en relación a los fechados, éstos han sido recuperados de las publicaciones donde son presentados técnicamente (en especial las que aparecieron en primer lugar en la revista Science y luego en Radiocarbon).

\section{1-LOS PROBLEMAS DE LA DATACIÓN}

A pesar de la existencia de diferentes manuales, notas y aproximaciones sobre el proceso de datación (Brothwell y Higgs, 1963; Aitken, 1974; Renfrew, 1979; Lumbreras, 1981; Mook et al., 1985; Garanger, 1992; etc.), uno de los problemas básicos que enfrenta la datación absoluta en la arqueología andina, especialmente peruana, es la ligereza y poca rigurosidad con la que se tratan los datos radiocarbónicos. Es por eso que nos vemos obligados a hacer una breve presentación de los principios y premisas del método de datación radiocarbónica y de su manejo, dirigido especialmente a los estudiantes y jóvenes arqueblogos que no tienen a su alcance las fuentes bibliográficas básicas para entenderlo.

\section{a) Las bases de la datación radiocarbónica}

Hacia fines de la década de los 40, Willard Frank Libby descubrió el proceso del ${ }^{14} \mathrm{C}$ y utiliz6, en sus primeros análisis, varias muestras provenientes del Perú, las cuales fueron suministradas por J. Bird (Amold y Libby, 1951: 119; Bird, 1985: 51).

El principio de la datación sostiene que nuestro planeta está sometido a un constante bombardeo de rayos cósmicos. Si bien la mayor parte de estos rayos son desviados por el campo magnético terrestre, una parte de ellos llega a atravesar la atmosfera. Estos rayos están constituidos por numerosas partículas que al entrar en la alta átmósfera, disminuyen su velocidad por el 
choque con otras partículas, lo cual origina la presencia de neutrones "lentos". Estos neutrones "lentos" entran en colisión con núcleos de átomos de nitrógeno ${ }^{14} \mathrm{~N}$, modificando así su constitución interna. De esta colisión se tiene como resultado la creación de un núcleo de carbono ${ }^{14} \mathrm{C}$ (Evin, 1987: 1042 - 1043). Este proceso puede ser expresado de la siguiente manera:

$$
{ }_{7}^{14} \mathrm{~N}+{ }_{0}^{1} \mathrm{n} \Rightarrow{ }_{6}^{14} \mathrm{C}+{ }_{1}^{1} \mathrm{H}
$$

donde: ${ }_{7}^{14} \mathrm{~N}$ es el nitrógeno atmosférico,

$$
\text { in es un neutrón producto de los rayos cósmicos, }
$$$$
{ }_{6}^{14} \mathrm{C} \text { es el radiocarbono y }{ }_{1}^{1} \mathrm{H} \text { es un protón }
$$

De esta manera, alrededor de 2 átomos de ${ }^{14} \mathrm{C}$ son producidos por segundo y por $\mathrm{cm}^{2}$ de superficie terrestre (Olive, 1988: 1). Sin embargo, este nuevo átomo de ${ }^{14} \mathrm{C}$ se oxida rápidamente en gas carbónico, debido a la importante cantidad de ozono $\mathrm{O}_{3}$ presente en la alta atmósfera. Dicho gas carbónico desciende y se mezcla rápidamente con el $\mathrm{CO}_{2}$ del aire de las capas bajas de la atmósfera (Evin, 1987: 1043). Sin embargo, debido a que el ${ }^{14} \mathrm{C}$ es un isótopo radioactivo, sus átomos se desintegran espontáneamente produciendo nitrógeno y liberando un electrón. Por lo tanto, se produce un proceso de formación y de desintegración que se conjuga y genera un equilibrio radioactivo, es decir, se mantiene constante la radioactividad del gas carbónico atmosférico (Delibrias, 1989 : 208),

Así pues, diluido en el aire, la mayor parte del carbono se intercambia sobre las grandes superficies de aguas marinas, pasando a formar parte de los organismos marinos (Evin, 1987: 1043). El resto, gracias al proceso de fotosíntesis, llega a formar parte de las células vegetales que son absorbidas (generalmente comidas), directa o indirectamente, por otros seres vivientes. Es así que, íntimamente ligado a otros dos isótopos de carbono estable: el ${ }^{12} \mathrm{C}$ y el ${ }^{13} \mathrm{C}$, el ${ }^{14} \mathrm{C}$ entra en la composición de todas las materias orgánicas y de todos los precipitados de carbonato de calcio (Ibid.: 1044) (Fig. 1).

Una vez que el intercambio de gas carbónico entre el ser vivo y la atmósfera cesa, es decir, a la muerte del organismo, el ${ }^{14} \mathrm{C}$ no es renovado y la concentración de este iś́topo disminuye a la mitad cada 5568 años (edad media propuesta por W. F.Libby), valor que ha sido adoptado desde 1951 (Delibrias, 1989 : 208). Ésto significa que si se mide la cantidad residual de radiocarbono se podrá deducir la edad de la muerte de la muestra carbónica. Así pues, será posible medir hasta 7 períodos de 5568 años, es decir, se podrán datar muestras de hasta 40000 años aproximadamente. Ejemplo:

\begin{tabular}{cl} 
Tiempo en años & $\%$ de ${ }^{14} \mathrm{C}$ \\
0 & 100 \\
5568 & 50 \\
11136 & 25 \\
16704 & 12.50 \\
22272 & 6.25 \\
27840 & 3.12 \\
33408 & 1.56 \\
38976 & 0.78 \\
\hline & $-\cdots$
\end{tabular}

El principio del método se basa en la hipótesis que la radioactividad natural del carbono ha sido siempre constante, al menos en los últimos 40000 años. Ésto implica también que la actividad ${ }^{14} \mathrm{C}$ es la misma en todos los organismos vivos. Finalmente, la hipótesis supone que la radioactividad del fósil fechado no ha sufrido, durante todo el tiempo de su preservación, ninguna modificación, salvo aquélla que se desprende del decrecimiento radioactivo (Delibrias, 1989: 208).

Para poder medir este decrecimiento, el método más usado es el del conteo, uno por uno, de los átomos del isótopo que se desintegran de una masa de muestra. Dicho conteo se puede hacer convirtiendo la muestra en gas, a lo que se denomina "datación en fase gaseosa" o por "conteo proporcional". También se le puede hacer convirtiendo la muestra en líquido, denominando entonces al proceso "conteo por espectómetro centelleante" o "conteo por centelleo líquido". En todos los casos, al momento de la desintegración, la liberación del electrón causa un fenómeno físico detectable, aunque debido a la presencia de otros electrones siempre habrá un "ruido de fondo".

La actividad actual del ${ }^{14} \mathrm{C}$ de la muestra fósil es medida y comparada con la actividad del carbono moderno de referencia, el cual es dado en forma de ácido oxálico por el National Bureau of Standards (NBS) de los Estados Unidos (Garanger, 1992: 144).

En los últimos años se ha desarrollado la datación ${ }^{14} \mathrm{C}$ en espectómetro de masa por acelerador de partículas (AMS), lo cual permite trabajar con algunos miligramos de carbono y en un tiempo menor (Valladas et. al., 1989: 211). Sin embargo, según Mook et al. (1985: 55 ), a nivel arqueológico pueden haber problemas con este proceso, debido a que una muestra pequeña puede tener un grado de asociación arqueológica reducido, o la muestra puede ser parte de un contexto no homogéneo. Su ventaja es que puede haber menos mezcla en el material destinado al fechado.

De esta manera se ha establecido un calendario ${ }^{14} \mathrm{C}$ (quiere decir en años basados en la escala ${ }^{14} \mathrm{C}$ ) a base 


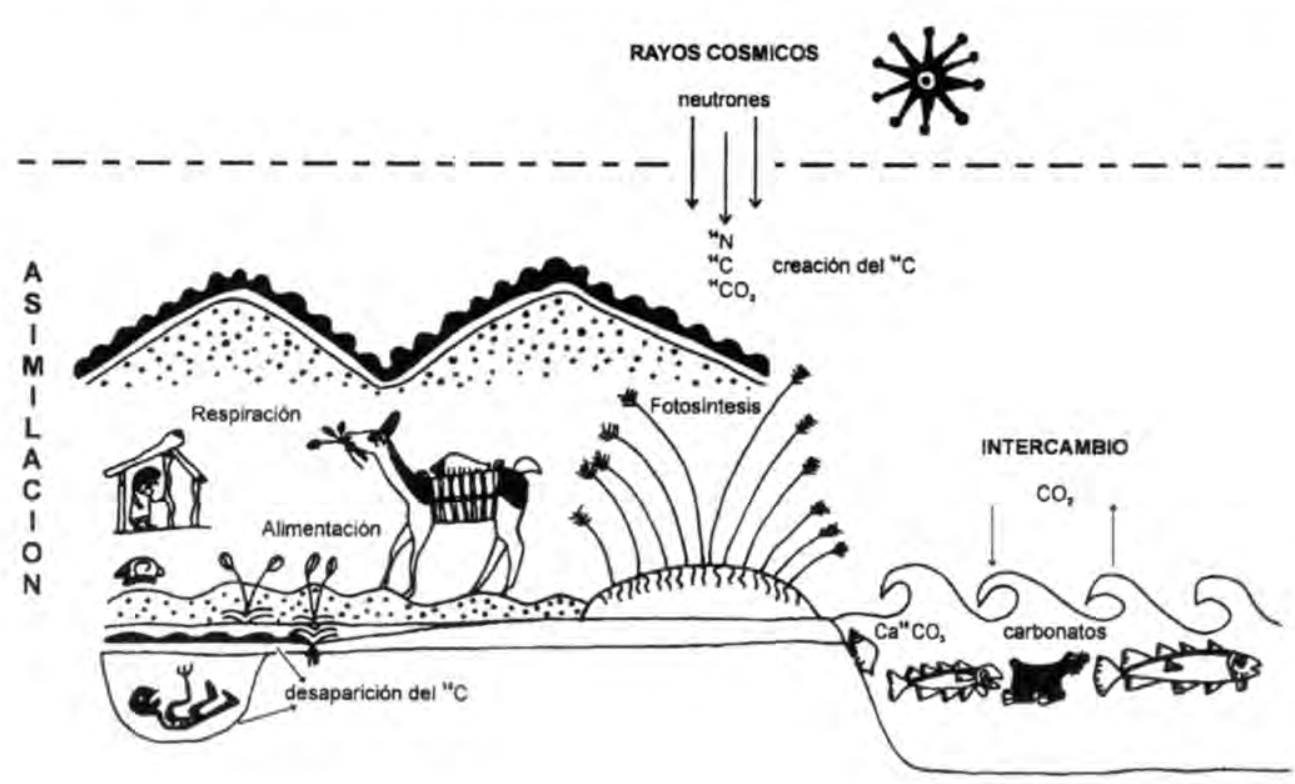

Fig, 1: El ciclo del C-14 en la naturaleza

de las miles de fechas obtenidas hasta el momento, y que toman como año $0 \mathrm{al}$ año solar (o real) 1950 después de Cristo (dicho año fue adoptado luego de la Conferencia de Groningen en 1959, ver Olsson, 1982: 38).

\section{b) Problemas que puede presentar una muestra} destinada al fechado

\section{Por su origen:}

- Las aguas profundas de los océanos presentan un intercambio lento con el $\mathrm{CO}_{2}$ atmosférico, lo cual les ocasiona una insuficiente renovación del ${ }^{14} \mathrm{C}$ que compense el decrecimiento radioactivo. Estas aguas profundas llegan a mezclarse con las superficiales, las cuales sí mantienen un intercambio rápido de carbono, dando como resultado un empobrecimiento del 5\% de ${ }^{14} \mathrm{C}$ en el agua superficial de los océanos, es decir, en 400 años, que es la edad media de la superficie de los océanos. Por lo tanto, las conchas marinas vivas, cuyo carbonato mantiene un equilibrio radioactivo con los carbonatos disueltos en el agua de mar, tendrán la misma edad aparente del agua misma. Por ello, las fechas BP de las muestras marinas necesitarán una corrección de + 400 años. Sin embargo, cada región ma-ina presenta un caso particular; así pues, en las regiones costeras, debido al afloramiento de las aguas profundas en estas zonas, tal corrección puede alcanzar valores entre 700 a 800 años (Delibrias, 1989: 209). En general, las fechas obtenidas de restos malacológicos no son recomendadas para su uso en arqueología.

En la tabla 1 se presentan los casos que han sido mayormente usados para el Formativo. Otras muestras aplicables al presente caso, que algunos arqueólogos podrían incluirlas en el Formativo y de las cuales no hemos obtenido mayores asociaciones arqueológicas, se muestran en la tabla 2.

- En los medios lacustres, los organismos acuáticos también han sido expuestos a diferentes concentraciones de la proporcion original de ${ }^{14} \mathrm{C}$ (Stuiver y Reimer, 1994: 9). En medios calcáreos, una muestra puede envejecer entre 3500 a 1300 años (Delibrias, 1989: 209). Los moluscos terrestres también pueden dar fechas muy antiguas, por lo que generalmente no se recomienda su uso en arqueología.

En nuestro conjunto de muestras no hemos encontrad, material que pueda presentar estos problemas.

- En la naturaleza existen procesos de enriquecimiento o de empobrecimiento de un iśtopo en relación a otro, lo que quiere decir que hay un fraccionamiento isotópico. En nuestro caso, el fraccionamiento isotópico tiene lugar entre el $\mathrm{CO}_{2}$ atmosférico y las plantas, luego del proceso de fotosíntesis. Así, el carbono de la celulosa en la mayoría de las plantas resulta empobrecido en $20 \%$ en ${ }^{13} \mathrm{C}$ en relación al $\mathrm{CO}_{2}$ atmosférico y el doble, es decir $4 \%$, en ${ }^{14} \mathrm{C}$ (Olive, 1988: 5). De este modo, por convención, todos los fechados deben estar normalizados en relación al $\partial^{13} \mathrm{C}$, en general en un valor de $-25 \%$. Se debe notar que plantas como el maíz (Zea mays) y gran parte de las Cyperáceas (Scirpus sp. por ejemplo) asimilan el $\mathrm{CO}_{2}$ siguiendo un proceso llamado $\mathrm{C} 4$, es decir un $\partial^{13} \mathrm{C}$ de alrededor $-12.5 \%$ (ver por ejemplo Burger y Van Der Merwe, 1990: 88-89), lo que corresponde a un 


\section{TABLA 1}

Fechados ${ }^{14} \mathrm{C}$ obtenidos sobre restos malacológicos

Algunos casos frecuentemente asociados al Período Formativo

\begin{tabular}{|c|c|c|c|c|c|}
\hline $\mathrm{N}^{0}$ muestra & Fecha BP & Sitio & Asociación & Excavó & Bibliografía \\
\hline C. 315 & $3572 \pm 220$ & Huaca Prieta & $\begin{array}{l}\text { Nivel HP 3-M } \\
\text { (precerámico con } \\
\text { agricultura) }\end{array}$ & J. Bird & $\begin{array}{l}\text { Arnold y Libby, } \\
\text { 1951: 119; Bird, } \\
\text { 1985: } 55 \text {. }\end{array}$ \\
\hline$L-122 B$ & $\begin{array}{l}5650 \pm 200 \\
6000 \pm 400\end{array}$ & Huaca Negra & "Middle Guañape" & W. D.Strong & $\begin{array}{l}\text { Kulp et.al., 1952: } \\
409-410\end{array}$ \\
\hline $\mathrm{L}-122 \mathrm{D}$ & $4300 \pm 200$ & Huaca Negra & "Upper Early Guañape" & W. D.Strong & Ibid. \\
\hline $\mathrm{L} \cdot 122 \mathrm{G}$ & $5300 \pm 200$ & Huaca Negra & "Lower Guañape" & W. D.Strong & Ibid. \\
\hline UCLA - $729 \mathrm{~A}$ & $3400 \pm 100$ & Sechin Alto & Nivel 5 & D. Collier & $\begin{array}{l}\text { R. Berger et al., } \\
\text { 1965: } 347 \text {. }\end{array}$ \\
\hline UCLA-729 B & $2640 \pm 90$ & Sechin Alto & Nivel 7 , más profundo que 5 & D. Collier & Ibid. \\
\hline $\mathrm{Ny}-224$ & $4070 \pm 90$ & $\begin{array}{l}\text { Loma de los Cardos } \\
(12 b-V I I-6510)\end{array}$ & $\begin{array}{l}\text { Nivel 100. Capa de detritus } \\
\text { cubriendo una choza }\end{array}$ & F. Engel & $\begin{array}{l}\text { P. Richard et al., } \\
\text { 1978: } 63\end{array}$ \\
\hline $\mathrm{Ny}-227$ & $3340 \pm 80$ & $\begin{array}{l}\text { Loma Huarangal } \\
(12 \mathrm{~b}-\mathrm{VII}-8570)\end{array}$ & $\begin{array}{l}\text { Nivel 100. De una capa de } \\
\text { desechos que cubria casas } \\
\text { de piedra. }\end{array}$ & F. Engel & Ibid. \\
\hline $\mathrm{Ny}-229$ & $2530 \pm 60$ & $\begin{array}{l}\text { Loma de Camotillo } \\
(12 b-\text { VII 945) }\end{array}$ & Nivel 1, cerámica "Chavín" & F. Engel & Ibid. \\
\hline $\mathrm{Ny}-228$ & $2130 \pm 70$ & $\begin{array}{l}\text { Loma de la Paloma } \\
(12 b-V I I 6048)\end{array}$ & $\begin{array}{l}\text { Nivel } 400 \text {, cerámica Lapa } \\
\text { Lapa }\end{array}$ & F. Engel & Ibid. \\
\hline Gif - 1072 & $2050 \pm 110$ & Chilca (12b - VII - 933) & $\begin{array}{l}\text { Nivel 1. Aldea de piedras } \\
\text { en Lomas. }\end{array}$ & F. Engel & $\begin{array}{l}\text { Delibrias et al., } \\
\text { 1971: } 225\end{array}$ \\
\hline
\end{tabular}

\section{Observaciones:}

C - 315, se trata de uno de los primeros fechados obtenidos para el Perú por el mismo W. F. Libby, realizado en el "Institute for Nuclear Studies" de la universidad de Chicago (Amold y Libby, 1951: 119). Estratigráficamente a mayor profundidad que las muestras C - 362 (4044 $\pm 300 \mathrm{BP}), 318$ b (3550 $\pm 600 \mathrm{BP})$ y 321 (2966 $\pm 300 \mathrm{BP})$. De la misma capa y contexto se obtuvo la muestra de madera 316 (4380 $\pm 270 \mathrm{BP}$ ) (ver: Bird, 1985: 51-58).

$\mathrm{L}$ - $122 \mathrm{~B}$, (Con un promedio entre las dos de $5750 \pm 180 \mathrm{BP}$ ). Encontrada a una profundidad de $0.25-1.75 \mathrm{~m}$, junto con L-122 D y G fueron recogidas y ubicadas estratigráficamente en el valle de Virú. Estas muestras sivieron para empezar a inferir sobre la posibilidad de error (fechas más "viejas") en la datación de conchas (Kulp et al., 1952: 409 - 410). Collier en 1955 (Collier, 1955: 25) las eliminó de sus datos para establecer la secuencia de Guañape en Huaca Negra.

$\mathrm{L}-122 \mathrm{D}$, a una profundidad de $1.75-2.25 \mathrm{~m}$.

$\mathrm{L}-122 \mathrm{G}$, a una profundidad de $2.75-2.40 \mathrm{~m}$.

UCLA - 729 A, (obtenida sobre restos de Fissurella maxima y Concholepas peruviana) y UCLA - 729 B (obtenida sobre restos de Aulacomya ater, Semele flavescens y Crepidula dilatata), según el informe radiocarbónico (R. Berger et al., Ibid.). las fechas presentadas son opuestas a las supuestas edades basadas en la estratigrafia.

Ny -224, 227, 228 y 229, provenientes del valle de Chilca. 
TABLA 2

Fechados ${ }^{14} \mathrm{C}$ obtenidos sobre restos malacológicos. Algunos casos frecuentemente asociados al Periodo Formativo sin mayor contextualización

\begin{tabular}{|l|l|l|l|l|l|}
\hline N0 muestra & Fecha BP & Lugar & Asociación & Excavó & Bibliografía \\
\hline GrN - 5546 & $3655 \pm 45$ & $\begin{array}{l}\text { Ancón, El Tanque } \\
\text { (11B-Vill-153) }\end{array}$ & $\begin{array}{l}\text { En lo alto de un monticulo con } \\
\text { basural erosionado. Nivel 0 }\end{array}$ & F. Engel & Vogel et al., 1969 \\
\hline Wis - 325 & $3650 \pm 65$ & $\begin{array}{l}\text { Salinas de Otuma cerca } \\
\text { a la laguna de Salinillas }\end{array}$ & $\begin{array}{l}\text { Sitio de ocupación } \\
\text { resto de Pecten purpuratus. }\end{array}$ & N. Psuty & $\begin{array}{l}\text { Bender et al., } \\
1970: 344\end{array}$ \\
\hline VRI - 121 & $3460 \pm 90$ & $\begin{array}{l}\text { Otuma, cerca de las } \\
\text { salinas }\end{array}$ & $\begin{array}{l}\text { Conchal de Pecten } \\
\text { Pecten a 50 cm de profundid. }\end{array}$ & $\begin{array}{l}\text { H. Kinzl - Universi- } \\
\text { dad de Innsbruck }\end{array}$ & $\begin{array}{l}\text { H. Felber, 1970: } \\
316\end{array}$ \\
\hline GX - 219 & $2785 \pm 75$ & $\begin{array}{l}\text { Arroyo seco, Km. 127 } \\
\text { carretera Panamericana } \\
\text { Sur }\end{array}$ & $\begin{array}{l}\text { Conchal sin cerámica ni } \\
\text { algodón. }\end{array}$ & F. Engel & $\begin{array}{l}\text { Engel en: Krueger } \\
\text { et al., 1966: 155 }\end{array}$ \\
\hline I-1340 & $2360 \pm 215$ & Cabezas Largas & $\begin{array}{l}\text { Estructuras con cerámica } \\
\text { Proto-Nasca. Restos Je Agas }\end{array}$ & F. Engel & $\begin{array}{l}\text { Trautman et al., } \\
1966: 199\end{array}$ \\
\hline
\end{tabular}

rejuvenecimiento del orden de 150 años (Delibrias, 1989: 209). Las conchas marinas tienen un $\partial^{13} \mathrm{C}$ de alrededor de 0\%00 (Delibrias 1989:209), lo que puede significar un error de -400 años. Otros valores para otros materiales son: plantas marinas: $-12 \%$; plantas de agua dulce: $16 \%$; plantas suculentas del desierto: $-13 \%$ y colágeno de hueso: $-19 \% 00$ (Bowman, 1990:23).

Los animales, incluyendo al hombre, pueden reflejar estos valores equivalentes de acuerdo al tipo de alimentación predominante, de ahí que un vertebrado que tuvo una alimentación predominantemente marina tendrá un valor $\partial^{13} \mathrm{C}$ distinto a lo normal.

La literatura antigua -que en gran medida incluye la mayoría de fechados obtenidos para el Perú- y actualmente algunos laboratorios, no presentan los fechados con una normalización por el $\partial^{13} \mathrm{C}$ precisa, es decir, de acuerdo a los materiales fechados, lo cual puede implicar un error de 400 años y más (Stuiver y Reimer, 1994: 10). Generalmente, como hemos sostenido líneas arriba, los laboratorios aplican una normalización general de - $25 \%$. En el caso del Formativo, se deberá tomar con mucho cuidado la mayoría de fechados "en bruto" que han sido obtenidos antes de la década del 60 , los cuales pueden no poseer ningún tipo de normalización.

Existen laboratorios que no han medido sistemáticamente el "fraccionamiento" y que lo hicieron solamente a pedido del cliente. Por ejemplo, Isotopes Incs. (Trautman y Willis, 1966:161) presenta esta remarca en el informe donde aparecen fechas de Chilca (Ibid:193): entre otras, I-812, 2420 \pm 175 (Aldea 1, nivel con cerámica chavinoide) y de Las Colinas, Ancón: I-810, 3775 2220. Otro caso es el del laboratorio de Groningen, el cual sólo tomó en cuenta, para algunas muestras, este error de normalización. En todo caso, la muestra Gro-618, $1765 \pm 155$ de Paracas, no presenta ninguna remarca en torno a su normalización.

Otros problemas similares existen para los fechados determinantes de la cronología del Formativo. Por ejemplo, Burger (1979: 154) informa que sus fechas de Chavín (ISGS - 506, -507, -486, -493 y UCR - 693, -694, $-705,-747$ y -748) no presentan correcciones por fraccionamiento isotópico. Según el informe de la Universidad de Illinois, con relación a estos fechados (Li Liu et al., 1986: 107), la correlación hecha por fraccionamiento isotópico empeź a ser incluida en sus fechados a partir de diciembre de 1979.

- Debido a una mezcla gaseosa incompleta entre los hemisferios, las muestras atmosféricas provenientes del hemisferio sur, producidas antes de 1850 (revolución industrial) c'eben ser sometidas a una reducción de $\mathbf{4 0}$ años (Vogel et. al., 1993: 74; Stuiver y Reimer, 1994: 10). Aparentemente, esta corrección todavía no ha sido hecha en los fechados peruanos debido a su reciente establecimiento y publicación (Ibid). En todo caso, ella debe ser realizada para calibrar las fechas.

-Se han registrado rejuvenecimientos de hasta 800 años en muestras correspondientes del VI al IV milenio antes de J. C., lo cual se explicaría por una disminución del campo magnético terrestre, que ocasionó un aporte de 10\% de ${ }^{14} \mathrm{C}$ (Olive, 1988: 4; Evin, 1989: 214; Damon et. al., 1986: 266). También hay fluctuaciones en el ${ }^{14} \mathrm{C}$, durante períodos breves, debido a variaciones en la actividad solar. Incluso, luego de períodos de enfriamiento terrestre, la concentración de ${ }^{14} \mathrm{C}$ deviene 
más elevada debido a que el $\mathrm{CO}_{2}$ baja en contenido (Olive, 1988: 4; Damon et. al., 1986: 266).

El mayor problema causado por estas fluctuaciones de ${ }^{14} \mathrm{C}$, es que ellas fueron tan abundantes durante algunos períodos de tiempo que vuelven prácticamente inválidos de discusión algunos fechados, particularmente para el establecimiento de secuencias cronológicas "finas". Este problema en el caso del Formativo es importante pues, por ejemplo, entre los $400 \mathrm{BC}$ hasta los $760 \mathrm{BC}$, los márgenes de error o desviación son muy amplios, tal como veremos más adelante en el procedimiento de calibración, que sirve para resolver este problema.

\section{Por factores ajenos:}

- Una muestra puede envejecer debido a la "contaminación", es decir, al aporte de carbono antiguo (Ibid.), por ejemplo, debido a la filtración de aguas que aportan elementos calcáreos, es decir carbonatos, que suministran edades antiguas. Incluso la capa misma puede poseer materias orgánicas fósiles (Mook et al, 1985: 29-30). La presencia de sólo $5 \%$ en peso de carbono antiguo puede envejecer una muestra hasta en 400 años, lo cual es peligroso en muestras recientes (Delibrias, 1989: 210).

Este caso puede haber sucedido con las muestras SI - 1210, SI - 1211, SI - 1212 y GX - 1127 , obtenidas por $\mathrm{H}$. Amat en los canales colectores de desagüe del templo de Chavín y que podrían haber sido carbonatadas tal como informa Burger (Burger, 1984: 278). Podemos inferir este problema en la presente discusión gracias a que conocemos las condiciones de asociación de la muestra (ver también Lumbreras, 1989: 114). Sin embargo, no sabemos de dónde provendrían exactamente los carbonatos llevados por el agua que debió circular por dichos canales de desagüe: ¿Del mortero que unía las piedras graníticas de construcción del templo?, ¿De las capas superiores?, ¿De la capa misma a la que pertenecía?, ¿O es, como dice Burger (Ibid.: 278), que los canales "...llevaron agua del río Mosna"?

Si queremos especular más con el problema, podemos preguntarnos si se trata de carbonatos que son comunes para todas las capas y depósitos. De presentarse este caso, significaría que los fechados podrían presentar distorsiones en su ubicación cronológica en todo el sitio. Esta pregunta no sería del todo descabellada, pues la región cordillerana de Ancash se caracteriza por tener fuentes termales y manantiales, los cuales son, por naturaleza, siempre ricos en carbonatos. Esto significaría que por simple acarreo, durante el período de lluvias, superficial o de la napa freática, de los carbonatos depositados naturalmente cerca de las vertientes, las muestras presentarían distorsiones casi sin excepción. Esta posibilidad se acrecienta inás si tomamos en cuenta que el sitio de Chavín y sus alrededores fueron afectados por un aluvión en 1945 (Lumbreras, 1989 : 17), que habría ocasionado la filtración de aguas superficiales, conteniendo carbonatos, hacia partes más profundas. Esperamos que algún día se hagan los análisis necesarios para aclarar este problema.

-Una muestra puede rejuvenecer por el aporte o "contaminación" de carbono reciente, debido al contacto con raíces de plantas o a la filtración de aguas con carbono reciente proveniente de capas de humus. En el caso de fechas recientes, $5 \%$ de carbono moderno representará un error de "sólo" 50 años (Delibrias, 1989: 210).

Este problema se puede presentar especialmente en las muestras provenientes de excavaciones que se encuentran cerca a campos de cultivo o en áreas donde hay abundante vegetación silvestre que crece o creció en algún momento.

Igualmente, la muestra puede ser contaminada debido a un mal almacenamiento, sea por mezcla con papel, algodón, etc.; por condiciones de humedad, o debido a preservantes aplicados por los actuales conservadores en los materiales arqueologicos, en los museos o en los sitios mismos.

-Hay muestras mixtas, de huesos humanos o animales con presencia de restos marinos, tales como: peces, moluscos, plancton, etc., y terrestres: granos, hierba, etc., los cuales presentan una mezcla de carbono marino y terrestre, que debe ser determinada para saber su influencia en la muestra (Stuiver y Reimer, 1994: 9 ).

Creemos que este aspecto debería ser considerado de importancia para las muestras que provienen de la Costa Peruana y que, generalmente, se encuentran mezcladas en los conocidos conchales o basurales que contienen, por lo tanto, abundancia de restos de origen marino. Hemos encontrado una muestra (N-86, 3810 \pm 160$)$ perteneciente al precerámico, Fase Gaviota (Patterson y Moseley, 1968; 128), que presenta en su descripción una "mezcla de plantas y arena" (Ravines y Álvarez, 1967: 25). En el informe del laboratorio para la serie de fechados publicados con N86 (Yamasiki, Hamada y Fujiyama, 1966: 324), se informa que las conchas fueron tratadas con $\mathrm{HCl}$; sin embargo, no sabemos el caso particular de esta muestra. Imaginamos que la arena fue separada, que los contaminantes fueron limpiados, y que se hicieron las correciones necesarias, tal como vienen haciendo los laboratorios japoneses desde muy temprano (ver por ejemplo Kigoshi, Tomikura y Endo, 1962: 84).

\section{c) Una muestra segura para fechar}

El arqueólogo debe de ser el primero en intentar extraer una muestra pulcra y bien registrada, apta para 
obtener un fechado válido. Es preciso eliminar todos los contaminantes visibles, tales como raíces y restos de calcáreo; evitar marcar la muestra y no ponerla en contacto con cenizas actuales, aserrín, algodón, papel, etc. Además, la muestra debe almacenarse en vidrio, plástico o aluminio, manteniéndola en un ambiente seco y oscuro (Mook et. al., 1985: 33). Es importante el registro detallado de la extracción de la muestra, en especial de los aspectos físicos, que permitan someterla a crítica para tomar con las debidas reservas el resultado del fechado.

Los laboratorios se encargan de tratar los materiales en los casos que hemos denominado de "contaminación", con ácido para eliminar los carbonatos y lavado con soda para eliminar rastros de humus. Estas técnicas dejan a la muestra en mejores condiciones para ser trabajada. Sin embargo, los contaminantes orgánicos son muy difíciles de remover (Mook et. al., 1985: 30). En el caso de las muestras de carbonatos provenientes de conchas, por ejemplo, se intenta lavar con ácido las partes superficiales que se supone pueden haber sido contaminadas. A pesar de esto, no hay ninguna evidencia que las contaminaciones profundas puedan ser eliminadas por lo cual resultan muestras poco recomendables (Evin, 1987: 1056-1057). Como en la mayor parte de casos, para las fechas obtenidas antiguamente, esta limpieza puede no ser muy válida.

Así pues, tenemos una serie de materiales para datar que pueden ser calificados, en relación a su grado de confiabilidad, por los resultados que pueden ser obtenidos sobre ellos. De esta manera, los utilizados principalmente en arqueología son:

Carbón de madera: es el mejor material para fechar mediante el ${ }^{14} \mathrm{C}$ debido a que es químicamente inerte, gracias a la combustión sufrida (Langouet et. al., 1992: 148). Si se trata de restos grandes de carbón, dicha muestra estará menos sujeta a contaminación, aunque bien podría haber intrusiones de raíces, por ejemplo. Sin embargo, diferentes carbones provenientes de un mismo árbol pueden dar fechas distintas: el centro de un árbol será siempre más viejo que su corteza periférica y las diferencias en las fechas serán grandes si se trata de árboles que viven mucho tiempo. Otro problema que pueden presentar los fechados es la reutilización de la madera a través del tiempo, en especial si se trata de grandes troncos.

Hueso: se trata igualmente de un buen material para fechar, siempre y cuando dicho fechado sea obtenido mediante la extracción del colágeno. Dicha operación es muy delicada y es un método que ha sido desarrollado solamente en los últimos años. De ser así, los fechados obtenidos son muy confiables. Sin embargo, hay que tener siempre en cuenta el medio en el cual estuvo enterrada la muestra (Aitken, 1990: 87; Evin, 1987: 1057), lo cual puede haber alterado su colágeno original.
De no seguirse el método de extracción del colágeno, los fechados obtenidos sobre muestras óseas generalmente son de mala calidad (Langouet et. al., 1992: 150; Evin, 1987: 1057), pues la base receptora es un buen absorbente de carbono contaminante.

Conchas: es el material menos recomendado para fechar, tal como ya hemos visto, por su origen así como por su difícil descontaminación, proporcionando fechas poco precisas. Aparentemente, las conchas de origen terrestre son menos precisas aún (Evin, 1987: 1057).

Aquí podemos detenernos para ver, una vez más, que las muestras provenientes del Templo de Chavín habrían podido acumular una serie de factores para su discordancia cronológica (Burger, 1984: 278, Lumbreras, 1989: 114).

En este caso, los huesos fechados podrían haber absorbido carbonatos debido a la disponibilidad del material óseo para absorber estos contaminantes, a lo cual se suma la fecha en la que fueron tratadas las muestras. Igualmente, la muestra GX-1127, hueso de camélido, ni siquiera puede ser, utilizada pues la cantidad de material analizado fue insuficiente, lo que no permitió siquiera obtuner el error sigma (Ziolkowski, 1994: 285).

\section{Muestras de la Galería de Las Rocas}

$\begin{array}{ll}\text { Si-1211 } & 3370 \pm 90(\text { Lumbreras, 1989: 114) y } \pm 190 \\ & \text { (Burger, 1984: 281) }\end{array}$

Si-1210 $\quad 3025 \pm 80$

Si-1212 2890 2125

GX-1127 3150土?

\section{Muestras de la Pirámide Tello}

Hemos encontrado otras muestras de Chavín, aparentemente obtenidas en 1982 por F. Kaufmann (Felber, 1984: 448), las cuales fueron obtenidas sobre huesos humanos (Ibid). El contexto indicado es de la pirámide Tello, al interior de las galerías (Ibid).

\section{VRI-8152290 \pm 90}

\section{VRI-8142060+90}

\section{d) Otros problemas}

- En cuanto a la fecha misma, su obtención es en base al conteo por minuto de pulsaciones o centelleos, producto de la liberación al azar de los electrones de la muestra. Su registro está regido por leyes estadísticas, lo cual incluye en el resultado una imprecisión debido a 
la liberación al azar de electrones y por el ruido de fondo, lo que se denomina desviación standard. La cifra así obtenida siempre deberá estar acompañada de un margen estadístico, representado por el símbolo " \pm ", que considere dicha desviación. Estadísticamente, una desviación standard o 1 sigma muestra una posibilidad de $68 \%$ de que la fecha obtenida sea cierta. Si se dobla esta desviación a 2 sigma, la posibilidad será de $95 \%$ y en 3 sigma la posibilidad será $99.8 \%$, lo cual significa que una interpretación errónea o abusiva de un primer resultado en 1 sigma será peligrosa si se quiere ser objetivo. Los laboratorios, por convención, dan sus resultados en 1 sigma (Evin, 1987 : 1048 - 1049). Igualmente, la utilización de fechados con un error muy grande debe ser solamente referencial, aunque pensamos que no es recomendable su utilización, debido a las imprecisiones que lleva consigo y lo arbitrario del uso, en general, de la fecha promedio.

Tenemos casos de fechas con errores tan amplios como en TK- $42,3900 \pm 900$, proveniente de Kotosh y asociada, probablemente, a la fase Mito (carbón de los fogones del templo ER-26, ver: Sato, Sato, Otomori y Suzuki, 1969: 514; ver también Bonnier, 1987: 901). Pensamos que un error \pm 100 a \pm 150 es el límite para una manipulación coherente en las secuencias, así como para su calibración.

- La imprecisión del fechado se acrecentará de acuerdo a la edad de la muestra. Así pues, mientras más vieja sea una fecha, su imprecisión será más amplia.

- Después de 1962 se observó que la edad media del ${ }^{14} \mathrm{C}$ que había obtenido Libby, $5568 \pm 30$, no era exacta y que el valor más exacto era de $5730 \pm 30$. Sin embargo, como hasta esa fecha ya se habían dado muchos resultados con el primer valor, se decidi6, en 1965, el continuar con la fecha "Libby", pues la diferencia no es significativa.

De cualquier forma, si se llega a manejar fechados que utilizaron el nuevo valor promedio de 5730, esto se puede solucionar dividiendo la datación con el factor 1.029 , producto de la división de $5730 / 5568$. Este nuevo valor, si se toma en cuenta para la discusión de fechas, ocasiona un incremento de edad del orden del 3 $\%$. Por ejemplo, una fecha de 1000 B.C. cambiará a una de 1090 B. C. (Aitken, 1974: 36).

- Todas las fechas radiocarbónicas en el mundo pueden ser comparadas entre sí; por eso podemos decir que hay un calendario ${ }^{14} \mathrm{C}$ y es por éso que los laboratorios dan sus fechados en B.P., tomando como base el año real de 1950. Como podemos deducir de los problemas citados anteriormente, el axioma del método ${ }^{14} \mathrm{C}$ que dice que "la radioactividad natural del carbono ha sido siempre constante, al menos los últimos 40000 años" no es necesariamente exacto, como lo podemos ver en las variaciones hacia el VI milenio, las fluctuaciones solares, etc. Por lo tanto, habrá siempre una desviación entre el calendario obtenido por el ${ }^{14} \mathrm{C}$ y el calendario solar o "real". Por ejemplo, en el VI milenio a.C., 1600 años del calendario ${ }^{14} \mathrm{C}$ representan 1000 años reales o solares (Evin, 1989: 214). Para poder superar, en particular, este problema, se ha desarrollado el método llamado calibración, que pasamos a enunciar brevemente.

\section{LACALIBRACIÓN}

Para poder conocer la desviación entre el calendario radiocarbónico y el real, es decir, para pasar del calendario ${ }^{14} \mathrm{C}$ al calendario solar o de años reales, es necesario cuantificar rigurosamente las fluctuaciones de ${ }^{14} \mathrm{C}$ en la atmósfera. Dicha cuantificación ha sido posible, en un principio, gracias a la comparación de las fechas obtenidas por el ${ }^{14} \mathrm{C}$ con los fechados dendrocronológicos.

Tenemos conocimiento que los trabajos en relación a la variación del ${ }^{14} \mathrm{C}$ y su respectiva calibración se remontan a 1958 y 1960 (ver: A. Birmingham, 1972: 151), que la primera curva de calibración publicada data del año 1966 y que, hacia 1982, este proceso ya estaba generalmente aceptado, gracias a que la calibración se basaba en la medida de la actividad del carbono 14 en los anillos de crecimiento de los árboles (Ver: Klein et al., 1982: 103).

Así se ha podido construir una secuencia cronológica bastante precisa, conocida como curva de calibración, que retrocede hasta 7890 años reales antes de Cristo, de acuerdo al programa de calibración llamado CALIB, versión 3.03, realizado por Stuiver y Reimer (1993), que utiliza los fechados carbónicos obtenidos de los anillos de crecimiento de árboles con edad conocida (Ver: Ibid. : 6).

Con la misma base que el proceso anteriormente descrito, pero con datos dendrocronológicos no necesariamente datados, esta secuencia ha sido alargada hasta los 9440 años reales antes de Cristo (Ibid. : 6).

Gracias a la comparación con fechas del método Uranium - Thorium (U - Th) y a medidas radiocarbónicas hechas en corales provenientes de Barbados, Mururoa y Las Galápagos (Bard et al., 1993: 191), la curva de calibración puede ir hasta 20000 años reales antes de Cristo (Ver también: Stuiver y Reimer, 1993: 223; Stuiver y Reimer, 1994: 6).

Las fluctuaciones del ${ }^{14} \mathrm{C}$ en la atmósfera son evidenciadas por la curva de calibración, la cual nos muestra periodos en los cuales una fecha radiocarbónica puede tener un rango de probabilidad muy amplio en años reales (figura 2). 


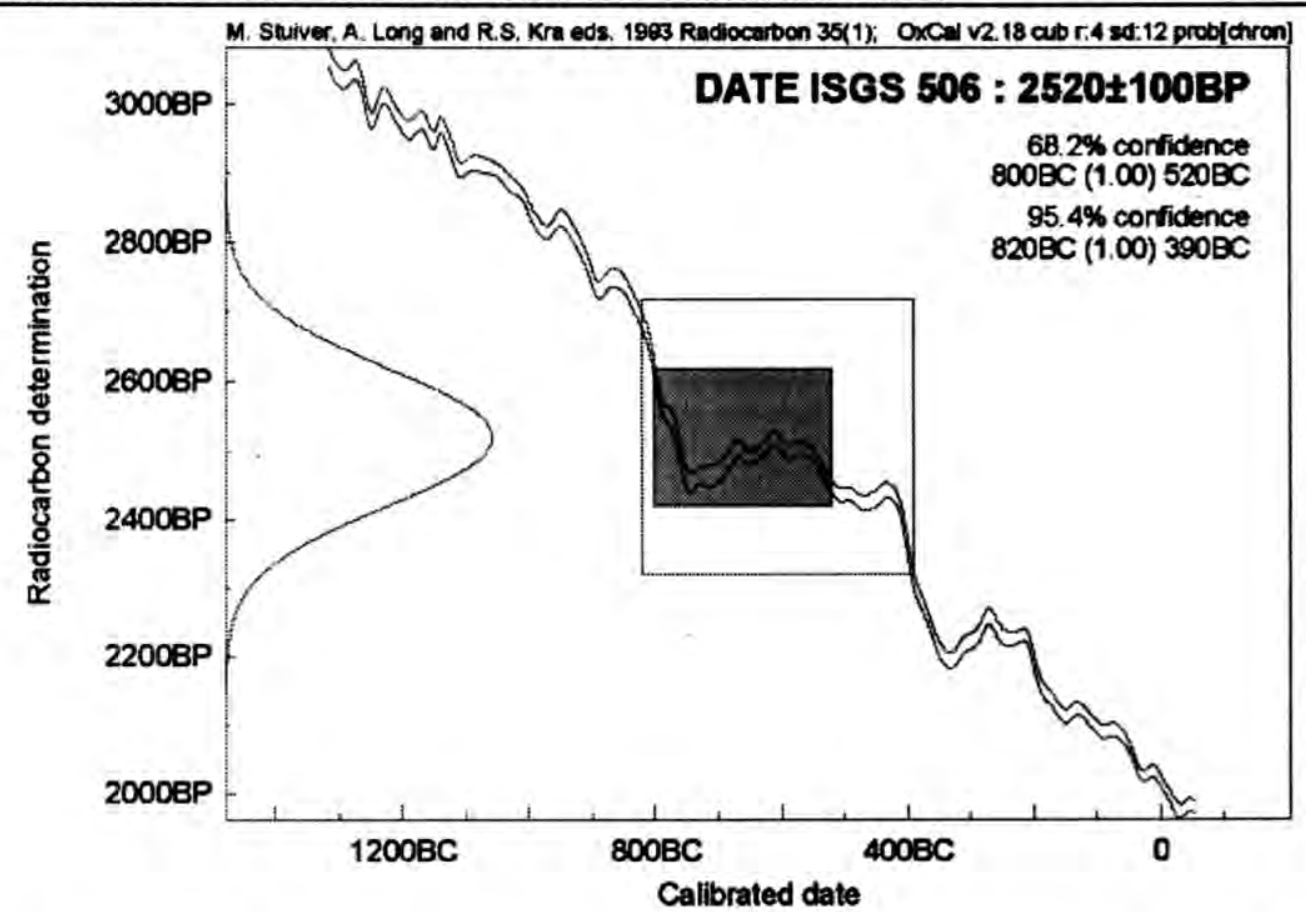

Fig. 2: Segmento de la Curva de Calibración en base a Radiocarbon 35 (1), editado por Stuiver, Long y Kra, 1993, en base al programa informático OxCal v. $2.18^{\text {(c) }}$ Ch. Bronk R. del Oxford Radiocarbon Accelarator Unit.

Este hecho es mostrado por un aplanamiento de la curva que, en nuestro caso, ocurre hacia aproximadamente $200 \mathrm{BC}$ a $330 \mathrm{BC}$ y entre $420 \mathrm{BC}$ a 750 $\mathrm{BC}$. Esto quiere decir que muchos de los fechados en discusión pertenecientes al Formativo Medio y Tardío, tendrían un gran rango de probabilidad, volviéndose imposible una discusión de tipo calendárico en torno a la duración de las fases a las cuales han sido asociados, al menos en términos puramente radiocronológicos. Aquí pensamos fundamentalmente en las fases Janabarriu, Ocucaje 3 y todas aquéllas que presentan fechados ${ }^{14} \mathrm{C}$ hacia los 2400 a $2500 \mathrm{BP}$.

\section{a) Problemas de la Calibración}

Para poder calibrar una muestra a base de CALIB ver. 3.03 , es necesario corregir algunos de los problemas vistos más arriba:

- Debido a los problemas con los océanos y las muestras lacustres, se hace necesaria una corrección de la influencia de los carbonatos en este tipo de muestras. Si bien la calibración para muestras marinas integra una corrección de tiempo dependiente del reservorio mundial de los océanos (Stuiver y Reimer, 1994: 9), es necesario acomodar en la calibración los efectos locales que ocurren en el proceso y que se expresa como la variable $\Delta R$. Para América del Sur, costa pacífica, tenemos valores de $\Delta \mathrm{R}$ de $5 \pm 50$ para la zona del Ecuador, obtenidos a base de muestras de conchas con edades históricas de la región que comprende Port Parker en Costa Rica, por el norte, y Guayaquil por el sur. Para la zona del Perú es de $190 \pm 40$, a base del mismo tipo de muestra que las obtenidas anteriormente, pero de la región que comprende el norte del Perú (ca. $10^{\circ} \mathrm{S} 80^{\circ} \mathrm{W}$ ) y Valparaíso en Chile (Stuiver et al., 1986: 1020; Stuiver y Braziunas, 1993: 156).

- Determinación del porcentaje del carbono marino en las muestras llamadas mixtas, aplicando el mismo proceso $\Delta R$ visto para las muestras marinas. (5730/5568).

- Corrección del período de vida media del ${ }^{14} \mathrm{C}$ hemisferio sur.

- Corrección de 40 años de las muestras del

- Normalización de la muestra por corrección del $\partial^{13} \mathrm{C}$ en caso de no haber sido sometida al menos a la corrección por convención de $-25 \% 00$ en el caso de las muestras vegetales (estos valores $\partial^{13} \mathrm{C}$ aparecen en Stuiver y Reimer, 1994: 36). Debemos anotar que según Olsson (1982: 39), algunas medidas viejas pueden ser descartadas o reevaluadas debido a medidas insatisfactorias del $\partial^{13} \mathrm{C}$.

Otro tipo de problema es el relacionado a la manera cómo son presentados los fechados por parte de los arqueólogos. Estos fechados generalmente no pueden ser utilizados correctamente o contextualizados por parte del investigador que pretende calibrar un conjunto de muestras, pues hay confusiones en el uso de términos, además de una mala presentación, como por ejemplo el código del laboratorio y el número de muestra. 
Es preocupante que siendo arqueólogos los que hacen los informes, éstos presenten los fechados sin ninguna asociación y contextualización que permitan relacionar las muestras con el conjunto arqueológico para definir, al menos, problemas físicos que influencian la datación y que derivan del origen de la muestra sin contar, lógicamente, con los problemas directamente arqueológicos de asociación estilística, tipológica, funcional, superposición, etc.

\section{b) Terminología a usar}

La terminología usada en la presentación de fechados está regida por convenciones internacionales. Por lo tanto, cualquier simbología o terminología que se use tiene sus parámetros teóricos y metodologicos, que definen cómo trabajar y determinar las muestras para su calibración o para cualquier intento de análisis. Sin embargo, el problema principal, que ya hemos nombrado en el párrafo anterior, está relacionado con la manera cómo son presentados los datos radiocarbónicos. El idioma usado puede ser también un problema en el caso de la literatura arqueológica peruana, pues mayormente se presenta en inglés. Además, debe precisarse el número de sigmas que se utiliza para el fechado. Por convención internacional los laboratorios dan los fechados en 1 sigma. Por lo tanto, pasamos a definir una lexicografía general:

Actualmente, en el medio científico que trabaja con fechados radiocronológicos, la presentación de una fecha BP (Before Present en inglés) o AP (Antes del Presente en español) significa que es una fecha ${ }^{14} \mathrm{C}$ BP convencional, es decir, normalizada por el $\partial^{13} \mathrm{C}$; pero si la encontramos con la mención incorrecta, quiere decir que no ha sido normalizada. Estas fechas BP pueden ser trabajadas normalmente, pues se mantienen en el calendario carbono 14, cuyo año 0 es 1950 . Es preferible trabajar con la denominación en inglés debido a las convenciones internacionales.

En este punto debemos detenernos para anotar que el término corregido o corrected ha sido usado, general y erróneamente, como sinónimo de calibración. También ha sido usado para indicar que la fecha había sido obtenida con una edad media de 5730 . El término no corregido o uncorrected ha sido empleado para las fechas obtenidas con el valor 5568 (ver: Neustupny, 1970: 44). Algunos arqueólogos usan el término uncorrected para presentar las fechas tal como han sido dadas por los laboratorios respectivos, pero en el calendario B.C. Como hemos visto páginas atrás, pueden haber muchas correcciones en la datación y si sumamos a ésto la gran variedad de términos usados para indicarlas, se dificulta saber si una fecha ha sido corregida o no, así como ponerla en el calendario AC - DC; o evaluar algún aspecto de ella, como el $\partial^{13} \mathrm{C}$, por ejemplo, según se entiende en los informes de la revista Radiocarbon; $u$ otros, como por ejemplo el período de vida 5568 como lo define Neustupny, o los 40 años de diferencia del hemisferio sur, etc.; o aún si ha sido calibrada simplemente (Ver este caso en Bird, 1985: 52-53; Pozorski, 1987: 17; Fung ,1988: 95-96; Burger,1988: 109).

Una fecha en A.C / D.C (BC / AD, Before Christ y Anno Domini o After Death, respectivamente), obtenida de un fechado radiocarbónico, significa que es una fecha ${ }^{14} \mathrm{C}$ BP no calibrada, de la cual se ha sustraido el valor 1950. En la actualidad, la presentación de este tipo de fecras no es recomendado debido a que no representa la realidad. Esta conversión dejó de ser usada oficialmente por los especialistas desde 1977, luego de la Novena Conferencia de Radiocarbón en 1976. En el caso de presentarse una fecha AC/DC, producto de un fechado ${ }^{14} \mathrm{C}$, ésta debe presentar la referencia de la curva de calibración usada para su conversión en años reales.

Esta simbología puede encontrarse en inglés, pero con letras minúsculas (b.p., a.d./ b.c., por ejemplo: Fung,1988: 95; ver la discusión de Chippindale, 1990: 186). Esta presentación fue propuesta por Glyn Daniel para la publicación de fechados radiocarbónicos en la revista "Antiquity", y sirve para representar las fechas que fueron obtenidas con una edad media de 5568 (G. Daniel, 1972: 265).

Las fechas Cal BC y Cal AD son fechas calibradas, es decir, son fechas que pertenecen al calendario de años reales o solares. Por convención internacional, los resultados de las calibraciones deben ser dados siempre en 2 sigma. Esto se debe a que las variaciones de la proporción de ${ }^{14} \mathrm{C}$ en la atmósfera no responden a leyes estadísticas, imposibilitando un cálculo de probabilidades uniforme para todos los fechados. Es por ésto también que, es preferible, en arqueología, no privilegiar ningún punto o fecha correspondiente al intervalo de 2 sigma.

Para unificar los diversos criterios que se usan en la literatura arqueológica andina, proponemos usar la terminología castellana que ha servido, en general, para determinar históricamente los años "reales" o "solares": a.C. (antes de Cristo o, si se quiere, antes de nuestra era) y d.C. (después de Cristo o de nuestra era), pero con la mención Cal. Esta medida servirá para poder aclarar a los científicos que quieren trabajar con ellas, la posición cronológica real y, al público interesado, tener la referencia siempre constante de los años calendáricos a base de los cuales vivimos. Está demás decir que la presentación del sigma se rige por convenciones internacionales.

Luego de esta revisión del fechamiento mediante el radiocarbono y su calibración, creemos que será posible, para los interesados, analizar, entender y 
criticar los fechados calibrados asignados al Formativo, publicados en los últimos 15 años. Entre los más conocidos tenemos: las fechas calibradas en Batán Grande, provenientes de estructuras quemadas asociadas a la ocupación formativa de Huaca Lucía - Chólope (Shimada et al., 1990: 21); las fechas calibradas pertenecientes a sitios del Período Inicial (Grieder et. al., 1988: 70, 71 y 72); las fechas radiocarbonicas provenientes de la Huaca de los Ídolos (Feldman, 1985: 77) y, finalmente, el listado de fechas radiocarbónicas asociadas al Formativo y calibradas, presentado en el libro de Burger (1992).

Un caso bien documentado corresponde a los fechados obtenidos en Huaca Prieta por Bird, y presentados en la última edición de los resultados de sus excavaciones, por J. Hyslop (Bird, 1985: 51-58). En este trabajo se presentan las fechas asociadas, así como las calibraciones respectivas, obtenidas a base de Ralph et al. (1973), Damon et al. (1974) y Klein et al. (1982), con su respectiva crítica.

\section{LAS INTERPRETACIONES}

a) Qué representa arqueológicamente una fecha radiocarbónica

En principio, los expertos en ${ }^{14} \mathrm{C}$, sean físicos, geólogos u otros especialistas, parecen estar de acuerdo en que "el ${ }^{14} \mathrm{C}$ permite datar la muerte del vegetal o del animal - que es usado como muestra de datación- y no un fenómeno histórico: la ocupación de un sitio, etc." (Olive, 1988: 5), de lo que se desprende que, en arqueología, para poder relacionar la fecha obtenida con el fenómeno histórico que se quiere fechar, dicha muestra debe haber sido recuperada con los métodos rigurosos de asociación que deben aplicarse durante las excavaciones arqueológicas. Además, para poder interpretar los problemas físicos de la muestra, es necesario conocer su tipo, rasgos de origen, influencias físicas o químicas ajenas, etc. Finalmente, siempre es necesario obtener varios fechados para poder corroborar los posibles problemas que afronta una muestra sola, y evidenciar los problemas que no pueden ser superados por el tratamiento de laboratorio, antes del procesado radiocarbónico de la muestra, ni por los cálculos necesarios antes de la calibración.

Todos estos aspectos que hemos discutido en torno a la problemática del Carbono 14 , casi nunca se establecen con precisión en la literatura arqueológica. Generalmente, debido a confusiones, desconocimiento, o simplemente por una manipulación abusiva de los datos obtenidos, los fechados son presentados sin considerar casi ninguno de estos aspectos, a pesar que se sabe que un fechado ${ }^{14} \mathrm{C}$ está sujeto a diferentes factores de error.
Otro aspecto que nos causa preocupación, como indicamos en la introducción del presente trabajo, es la constante discusión de la duración del Formativo y sus posibles fases. Se toma a los fechados radiocarbónicos como si éstos representaran años reales (años solares o históricos) con los cuales se pueden establecer períodos históricos. Con el artificio de restar 1950 a una fecha BP, los arqueólogos creemos convertirla en una fecha a.C., y así se inserta en el calendario real histórico. Tal como hemos visto, intentar sostener una discusión con esta presunción falsa es inútil, particularmente si se proponen fases con exactitud casi anual, como se hace en la actualidad.

Nos causa extrañeza que este tipo de presentacióı de datos continúe en esta última década, ya que la proposición de no correspondencia entre los años ${ }^{14} \mathrm{C}$ y los años solares, y el uso de calibraciones para intentar solucionar este problema se ha hecho desde hace casi tres décadas y, tal como vimos páginas atrás, el uso de las fechas a.C.- d.C. fue descontinuado hace más de 15 años, salvo si se indicaba una referencia de calibración. El hecho es más sorprendente cuando vemos que la calibración no ha sido aplicada por arquéblogos que tienen la oportunidad de acceder fácilmente a ella, sino solamente por aquellos que han producido bibliografía especializada o han hecho referencia al problema (Ver por ejemplo la primera parte de la obra de Renfrew [1979] (1983): La Revolution du Radiocarbone).

El problema del debate en torno a las dataciones, es que no se confrontan los fechados BP para su discusión y crítica, sino que se intenta validar un fechado por sí mismo, olvidando discutir los factores arqueológicos o físicos de las muestras, independientemente de sus asociaciones, superposición y contexto. Esto ha causado que, en general, la cronología histórica se vuelva dependiente de las fechas radiocarbónicas (Neustupny, 1970: 40).

\section{b) Los fechados, su presentación y asociaciones: algunos ejemplos}

Un caso que ejemplifica el mal uso de los datos es el de la muestra UCLA - 729 A, $3400 \pm 100$. Esta fecha ha sido utilizada como valedera, hasta los últimos años, para sustentar la relación de unos posibles frisos en Sechín Alto con el sitio Pampa de las Llamas - Moxeke y así mostrar una temprana ocupación del sitio de Sechín (ver S. Pozorski, 1987: 21). Primero, la discusión de la fecha se hace en años B.C. $(1450 \pm 100)$, aunque se dice que es una fecha no corregida (uncorrected $i$ ?). Segundo, en la publicación original, donde se puede obtener dicha fecha (Berger et al., 1965: 347), se remarca la condicion de la muestra, tal como haber sido obtenida de conchas Fisurella máxima y Concholepas peruviana, lo cual prácticamente la descarta como una muestra válida. A ésto se añaden fechas en contradicción con la 
estratigrafía, e incluso una fecha más joven (UCLA $729 \mathrm{~B}, 2640 \pm 90$ ), que proviene de un nivel más profundo que el de la muestra $729 \mathrm{~A}$, aunque obtenida también a base de conchas (véase la tabla 1). Nada de lo anterior se cita en el estudio mencionado.

Esta misma problemática se presentó cuando se quiso asociar las Fases Guañape Temprano y Medio de Virú con las de Valdivia A y B de la costa del Guayas, tomando como único medio de comparación las dos dataciones obtenidas sobre conchas en Virú (véase la tabla 1) - muestras L - 122B, y L- 122D -; que daban fechas antiguas, a pesar que existían otras más recientes sobre restos vegetales, en el mismo nivel, con las obtenidas en el Guayas, sobre conchas solamente. Sin embargo, esta relación fue criticada por la parcialidad en el manejo de los datos (Ver un resumen de esta discusión en Lanning, 1963: 220).

Debido al uso de literatura antigua, algunas veces las fechas son presentadas de manera inexacta. Es el caso de la muestra L-404 A, $2300 \pm 80 \mathrm{BP}$ (Olson y Broecker, 1959: 22). Esta fecha ha sido usada generalmente por los arqueólogos en años BC y sin referencia al código de laboratorio, con un valor de $342 \pm$ 80 BC (publicada así en Collier, 1962: 413). El problema aquí es que dicho valor en $\mathrm{BC}$ ha sido obtenido usando como año 0 a 1958 y no a 1950 pues, en la época de su primera publicación, la fecha 1950 todavía no estaba generalizada. En todo caso, debería ser una fecha BC de $350 \pm 80$. Posteriormente, se ha seguido usando la fecha de $342 \pm 80 \mathrm{BC}$, ocasionando problemas para su ubicación exacta, e incluso para convertirla en años BP se ha llegado a agregar, a esta fecha BC, el valor actual de 1950 años, lo que da una fecha de $2292 \pm 80$ BP (S. Pozorski, 1987: 17 y 27), agregando coñfusión a su tratamiento.

En cuanto a sus asociaciones, la muestra proviene de la madera de un dintel de la fortaleza de Chanquillo. Esta madera sería del género Pithecellobium $s p$., según el informe técnico de Olson y Brocker (Ibid.). Lamentablemente, no sabemos si esta muestra estaba cubierta o a la intemperie, siendo más probable lo último, conociendo el tipo de sitio que es Chanquillo (Ver el informe de los trabajos de superficie en Collier, 1962: 411-417). Así pues, esta muestra pudo haber sufrido alteraciones en su composición de ${ }^{14} \mathrm{C}$, aparte que el sitio pudo haber sido reutilizado posteriormente. La cerámica asociada, de superficie, es la que Collier (1962: 413-414) llamó estilo Patazca y que consideró como un estilo tardío del Período Chavín (Ibid.: 414). Otra fecha de Chanquillo es Gif-2482, 2070 \pm 100 , que fue recuperada encima de la entrada de la "fortaleza" (Ziolkowski, 1994: 265; S. Pozorski, 1987: 17).

Otro caso de utilización de datos antiguos es el de las muestras L - $335 \mathrm{C}, 1840 \pm 100$ y L $-335 \mathrm{D}, 1940 \pm$ 100 (Broecker y Kulp, 1957: 1329), que sirvieron para datar las fases Ocucaje 10 y 9 , respectivamente (Menzel et. al., 1964: 4). Si bien han sido presentadas con un buen nivel de asociación, dichas fechas han sido dadas en años $\mathrm{AD}$ con valores de 116 y 16 , respectivamente, usando como año 0 el año 1956 (ver también Menzel, 1971: 44 y cuadro I).

\section{c) Algunas calibraciones a base de CALIB ver. 3.03 y} sus consecuencias

Podemos empezar nuestros ejemplos con un caso que acabamos de citar, asumiendo sin mayores pruebas que su fechado sea correcto: el de la muestra UCLA 729A, $3400 \pm 100$ BP. Tratándose de un fechado obtenido sobre concha, es necesario calibrarlo con una curva de calibración marina, que toma en cuenta los diferentes problemas provenientes de los reservorios marinos. Aplicando $\Delta \mathrm{R} 190 \pm 40$ y la curva de calibración, el promedio de esta misma fecha avanza hasta cal BC 1038, que difiere bastante de $1450 \mathrm{BC}$, producto de la sola sustracción de 1950 .

Sin embargo, el rango de probabilidad en 2 sigma (95.4\% de seguridad), cae entre cal BC 768 hasta cal BC 1813. Es decir, con 1045 años de rango de probabilidad, este fechado puede pertenecer a una parte del Horizonte Temprano y a la totalidad del Período Inicial, si es que seguimos aceptando los límites cronológicos propuestos hasta el momento para estos periodos. Lo único que nos asegura este resultado es que el fechado pertenece al Período Formativo, si es que la muestra no fue contaminada en algún momento, cosa de la cual no podemos estar seguros. En general, debemos notar que las fechas promedio calibradas sobre conchas tienden a rejuvenecer. En el caso presente, más de 400 años.

Ya que nuestro interés particular es el Período Formativo, podemos usar algunos fechados que sirven para determinarlo cronológicamente, que se presentan bien asociados y representan áreas específicas. Esta selección es puramente arbitraria, pues tomaremos fechas que coinciden con los límites propuestos, y sólo nos servirá como un pretexto de discusión.

El límite inferior del Período Inicial, $1800 \mathrm{BC}$ (sic) o $3750 \mathrm{BP}$, ha sido determinado a base de los fechados publicados en Andes 2: Gak 262, 3800 \pm 110 BP (Izumi y Sono, 1963: 154; ver también Izumi en: Sato et al., 1969: 514), que posee una fecha casi similar en la muestra Gak $765,3780 \pm 90$ BP (ver Izumi en Sato et. al.:Ibid), siendo las dos fechas más antiguas de un conjunto de cinco, obtenidas de la fase más antigua con cerámica en el sitio de Kotosh, llamada Kotosh Wayrajirca. Las otras 3 fechas son: N-69-2, 3100 \pm 130 BP(Yamasaki et. al., 1966: 336); TK $-43,3200 \pm 80$ BP (Sato et. al.: Ibid)y TK - 108, $3000 \pm 80$ BP (Kobayashi et. al., 1974). 


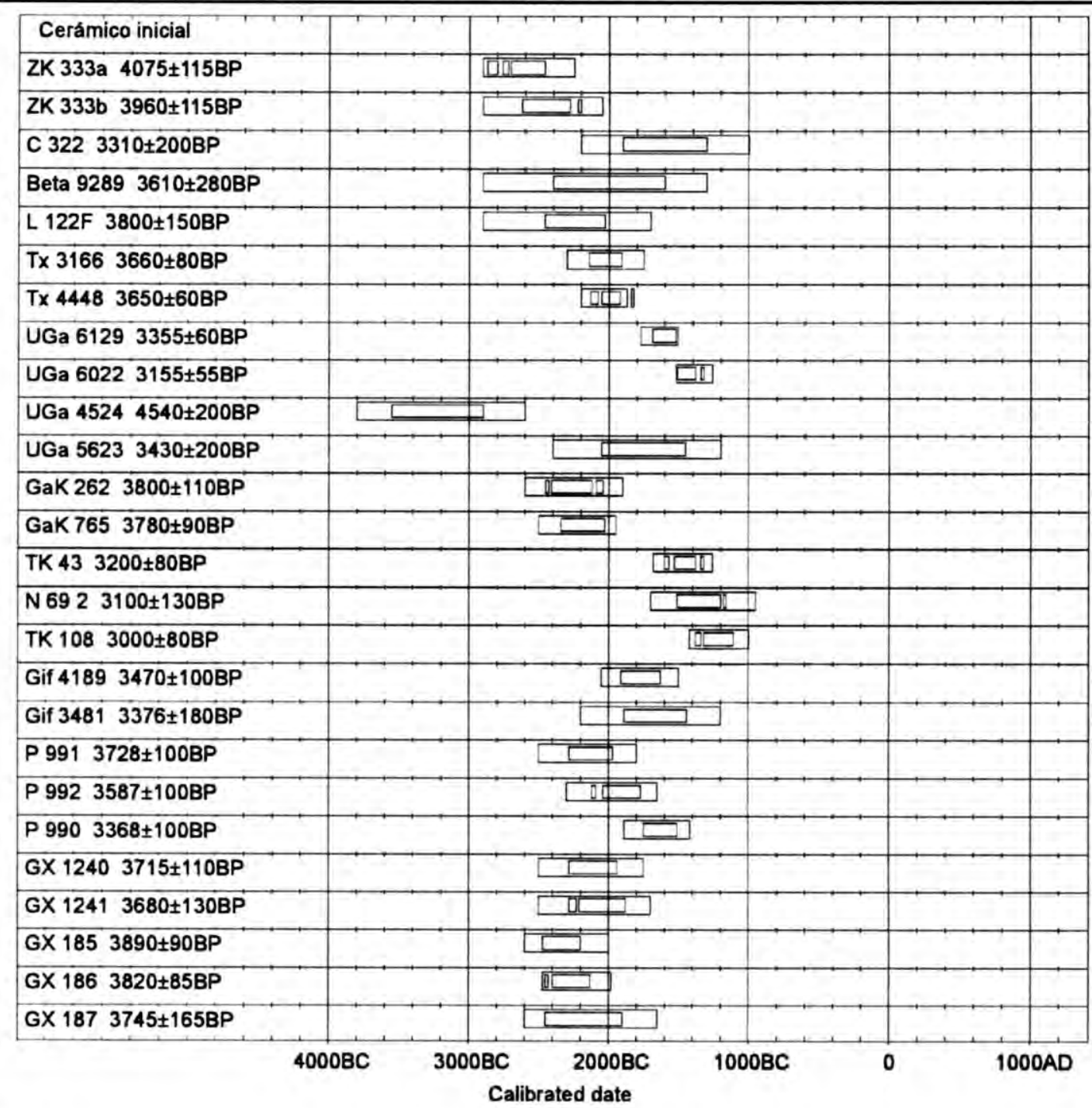

\section{Cuadro 1}

Fechas calibradas para el Período Cerámico Inicial a base de OxCal v. 2.18. Calibración sin ningún tipo de corrección. ZK-333a y b: Pandanche. C-322 y Beta-9289: Huaca Prieta. L-122F: Huaca Negra. Tx-3166 y Tx-4448: La Galgada. UGa 6129, 6022: Bahía Seca. UGa 4524, 5623: Tortugas. GaK 262 a TK-108: Kotosh Wayrajirca. Gif-4189, 3481: Telarmachay. P-991 a 990: Casa de la Tía. GX-1240, 1241: Ancón. GX-186, 187: Erizo y Mastodonte

Estas fechas reforzaron la ya propuesta para la cerámica temprana en la costa peruana, Guañape Inicial: L - 122 F, $3800 \pm 150 \mathrm{BP}$, proveniente del sitio de Huaca Negra (Kulp et, al., 1952: 410; Collier, 1955: 25). Otra fecha que ha servido como referente (Fung, 1982: 461) está asociada a Ancón Colinas: I - 810, $3775 \pm 220$ BP (Trautman et. al., 1966: 197-198). Igualmente, en La Galgada tenemos fechas como Tx $3166,3660 \pm 80 \mathrm{BP}$ y Tx 4448, $3650 \pm 60$ BP. En la costa sur, la Fase Erizo presenta dos fechados: GX - 185, $3890 \pm 90$ BP y GX 186, $3820 \pm 85$ BP (Fung, 1988: 96).

Como se puede apreciar en el cuadro 1, parece bastante probable un posible retroceso de las fechas aceptadas hasta el momento.
Si calibramos los fechados obtenidos para el límite reciente del Formativo sucede un fenómeno inverso al precedente: las fechas promedio tienden a ser mas jóvenes o presentan mayor probabilidad de serlo (véase el cuadro 2).

Las fases estilísticas de Ocucaje o Paracas (Menzel et. al., 1964) sirvieron como base para la división del Horizonte Temprano; así, la fase tardía del estilo Ocucaje, Ocucaje 10, Paracas Necrópolis o Topará, representaría el final del Horizonte Temprano contemporáneo, aproximadamente, con el final del Período Formativo.

Menzel et, al. (1964: 4) proponen que la fecha dada en la muestra L - 335 C, $1840 \pm 100$ BP (Broecker 


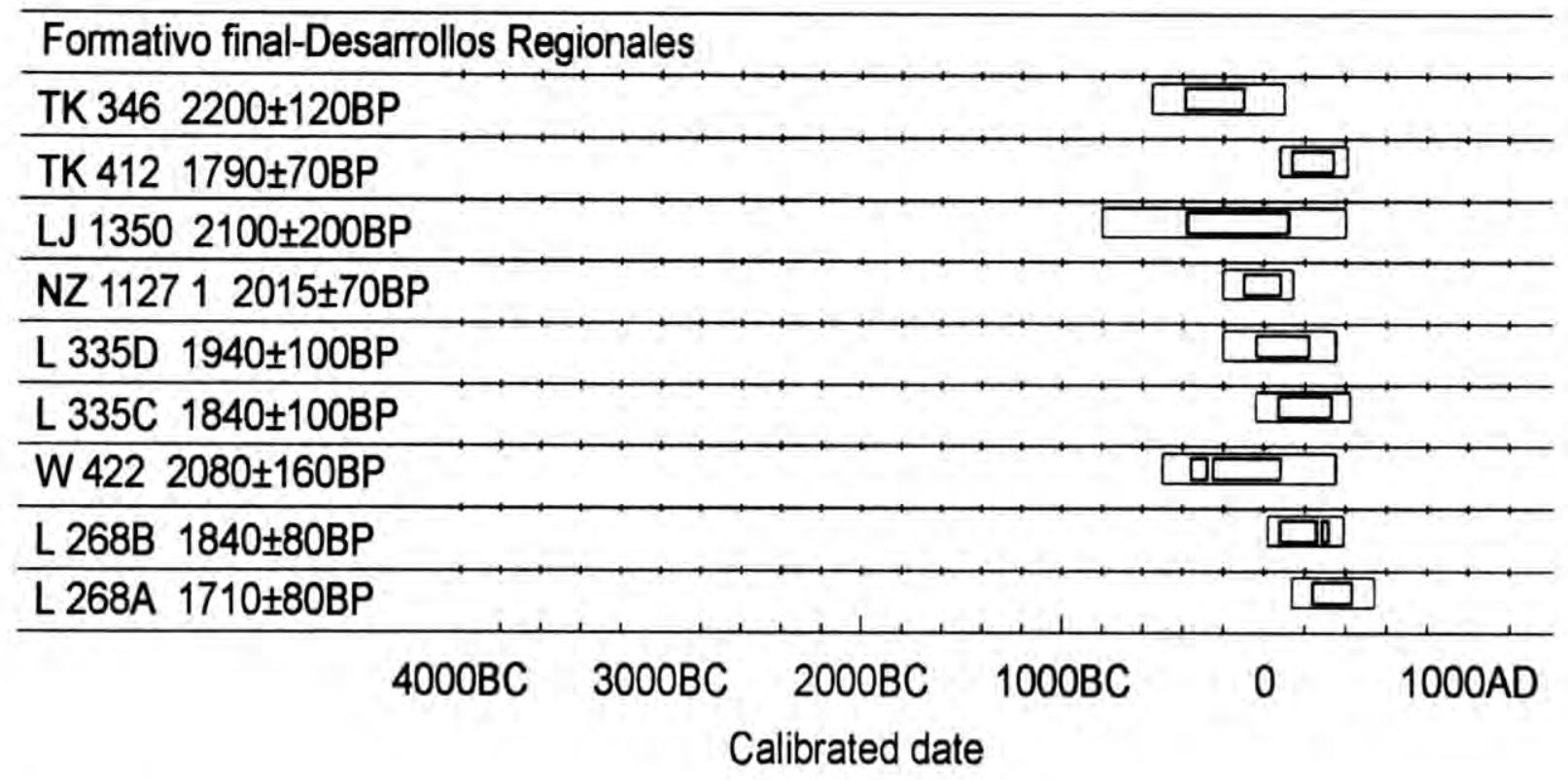

\section{Cuadro 2}

Fechas calibradas de la transición Formativo Tardío a Desarrollos Regionales a base de OxCal v. 2.18. Calibración sin ningún tipo de corrección.

TK-346, 412: Huacaloma. LJ-1350 a L-268A: Paracas.

y Kulp, 1957: 1329), está asociada a la Fase Ocucaje 10. Burger (1988: 109-110), a base de unas fechas obtenidas por Engel en Cabezas Largas, deja entender que el final de Paracas se asocia a la muestra NZ- 1127 2, $2060 \pm 170$ BP (Burger, Ibid.: 109). Si solamente tomamos estas dos fechas calibradas, el rango de probabilidad puede ir, aproximadamente, entre cal 400 $\mathrm{AD}$ hasta cal $500 \mathrm{BC}$.

Tomando como ejemplo el caso del Formativo Medio-Janabarriu-Ocucaje 3, conocido como "influencia Chavinoide", en la costa sur y las fases de Paracas, los márgenes de probabilidad son bastante amplios, en comparación a las proposiciones hechas sobre una posible extensión cronológica de esta influencia "chavinoide", de acuerdo al ejemplo de Burger (Ibid, 109-110).

\section{d) ¿Podemos confiar en los resultados de la calibración?}

La precisión de los métodos de calibración aumentará en los próximos años, y esperamos que el cuerpo de fechados radiocarbónicos se enriquezca. Por el momento, su calidad y cantidad es bastante débil para un período tan importante. El problema para la interpretación de la calibración de fechas que corresponden al Formativo Peruano, es que la amplitud de fechas obtenidas es bastante grande debido a las variaciones de la concentración de ${ }^{14} \mathrm{C}$ en la atmósfera. Es así que actualmente, en Europa, para los períodos de la Edad de Hierro (fases Hallstatt - 1200 a 500 a.C. - y La Tene - 400 a 50 a.C.), los arqueólogos prefieren utilizar métodos alternativos al ${ }^{14} \mathrm{C}$ (dendrocronología por ejemplo), debido al amplio margen de error demostrado por las técnicas de calibración.

A pesar de este gran inconveniente, que prácticamente terminaría por sepultar toda discusión en torno a las dataciones ${ }^{14} \mathrm{C}$ "en bruto" para el Período Formativo, por el momento el método de calibración puede servir, en algunos casos, como marco referencial cronológico, aunque de manera bastante gruesa.

Pensamos que, de esta manera, queda una vez más desvirtuada la idea que mediante una discusión puramente cronológica, es posible tratar arqueológicamente el pruceso cultural prehispánico. Dicho nivel de debate quedó negado ya en los años 50 , antes del uso generalizado de la datación ${ }^{14} \mathrm{C}$, cuando gracias al proyecto Virú, se mostró que la arqueología en el Perú podía ser algo más que una secuencia de fases. Una vez más vemos que la arqueología debe de ser entendida como una ciencia rigurosa, como proponía Childe en los años 30 , que necesita de principios básicos para poder interpretar y ubicar procesos de tipo universal, por ejemplo cronológicamente, como los que ocasionaron la presencia de un período llamado Formativo en los Andes Centrales.

Finalmente, esperamos que se inicie pronto un debate sobre la contextualización de los fechados 
arqueológicos de los Andes Centrales, con una amplia participación, tanto de los arqueólogos responsables de los distintos materiales, así como de la mayoría de responsables de laboratorios, para poder desentrañar las gran cantidad de incógnitas existentes en torno a los fechados ${ }^{14} \mathrm{C}$.

\section{Agradecimientos:}

Quiero agradecer al Dr. M. Ziolkowski por haberme facilitado el acceso a su base de datos "Andes", así como a P. Y. Nicod del Departamento de Antropología y de Ecología de la Universidad de Ginebra. Finalmente a A. Chu Barrera, por la paciencia en la recepción del presente texto. A ellos mil gracias.

\section{BIBLIOGRAFIA}

AITKEN, M.J.

1974 Physics and Archaeology. Oxford University Press. Londres.

\section{ARNOLD, J.R. y W.F. LIBBY}

1951 "Radiocarbon Dates". En: Science, Vol. 113, $N^{\circ} 2927$, pp. $111-120$.

BARD, Edwuard, Maurice ARNOLD, Richard G.FAIRBANKS y Bruno Hamelin

$1993 \quad{ }^{230} \mathrm{Th}-{ }^{234} \mathrm{U}$ and ${ }^{14} \mathrm{C}$ Ages Obtained by Mass Spectrometry on corals". En: Radiocarbon, Vol. 35, N 1, pp. 191 - 199.

BENDER, H, ReidA. BRYSON y David A. BAERRELS

1970 "University of Wisconsin Radiocarbon Dates VII". En: Radiocarbon, Vol. 12, N 1 , pp. 335-345.

BERGER, Rainer y W. F. LIBBY

1966 "UCLA Radiocarbon Dates V". En: Radiocarbon, Vol. 8, pp. 467 - 497.

BERGER, Reiner, G. J. FERGUSONy W.F. LIBBY

1965 "UCLA Radiocarbon Dates IV". En: Radiocarbon, Vol. 7, pp. 336 - 371 .

BIRD, Junius

1985 "The Preceramic Excavations at the Huaca Prieta Chicama Valley, Perú". John Hyslop editor. En: Anthropological Papers of the American Museum of Natural History, Vol 62, part 1. New York.

BIRMINGHAM, A.

1972 "The history of C14 Calibration". En : Antiquity. Vol. 46, № 182, pp. 151 - 152.

BOWMAN, Sheridan

1990 Radiocarbon Dating. British Museum Publications, Londres.
BROECKER , W.S. y KULP, J. L.

1957 "Lamont Natural Radiocarbon Measurements". En: Science. Vol. 126, $\mathrm{N}^{\circ}$ 3287 , pp. $1324-1334$.

BROTHWELL, Don y Eric HIGGS (edit.)

1963 Science in Archaeology. T. and H. New York.

BURGER, Richand

1979 "Resultados preliminares de excavaciones en los distritos de Chavín de Huántar y San Marcos, Perú". En: Arqueología Peruana: Investigaciones arqueológicas en el Perú, 1976. Seminario. R. MATOS compilador. pp. 133 - 155. Lima.

1984 "The Prehistoric Occupation of Chavín de Huántar, Perú". En: University of California Publications in Anthropology. Vol. 14. University of California Press.

1988 "Unity and heterogeneity within the Chavin horizon". En: Peruvian Prehistory. Richard Keatinge editor, pp. 99 - 144. Cambridge University Press.

BURGER, Richard y Nikolaas J. van der Merwe

1990 "Maize and the Origin of Highland Chavín Civilization: An Isotopic Perspective". En American Anthropologist. Vol. 92, $\mathrm{N}^{\circ} 1$, marzo. pp. 85 - 95 .

\section{COLLIER, Donald}

1955 "Cultural Chronology and Change as reflected in the ceramics of the Viru valley, Peru". En: Fieldiana: Anthropology. Vol. 43.

1962 "Archaeological Investigations in the Casma Valley, Peru". En: Akten des 34 International Amerikanistenkongresses. Wien, $18-25$. juli 1960. pp. $411-417$. Verlag Ferdinand Berger, Horn - Wien.

\section{CHIPPINDALE, Christopher}

1990 "How Many Kinds of Age do Historical Scientists need, and what should they be called?" En: MOOK y WATERBOLK editores, "Proceedings of the Second International Symposium 14C and Archaeology - Groningen, 1987", PACT, N²9, pp. 183 - 194.

DAMON, Paul y Timothy W. LINNICK

1986 "Geomagnetic-Heliomagnetic Modulation of Atmospheric Radiocarbon Production". En: Radiocarbon, Vol. 28, N²A, pp. 266 - 278.

DAMON, Paul, Charles FERGUSON, Austin LONG y E. I. WALLICK

1974 "Dendrochronologic Calibration of the Radiocarbon Time Scale" American Antiquity. 39. pp. 350 - 366 . 


\author{
DANIEL, Glyn \\ 1972 "Editorial", En: Antiquity. Vol. 46, N 184, \\ pp. 265.
}

DELIBRIAS, Georgette

1989 "Datation par le Carbone 14". En: Le Temps de la Préhistoire. 208 - 210 . Dijon. Societé Préhistorique Française.

DELIBRIAS, Georgette, M.GUILLIER y J. LABEYRIE 1971 "Gif Natural Radiocarbon Measurements VI" Radiocarbon, Vol. 13, №2, pp. 213 - 254.

EVIN, Jacques.

1986 "Les Méthodes de Datation". En : La Préhistoire, d'un Continent à l'Autre. 21 - 30. Paris. Editions Larousse.

1987 "Carbone 14". En: Géologie de la Préhistoire :Méthodes, Techniques, Applications. 1041 1060. Paris. AEEGP.

1989 "Temps Carbone 14 et Temps reel par la correction dendrochronologique des dates carbone 14". En : Le Temps de la Préhistoire. 213 - 217. Dijon. SPF.

FELBER, Heinz

$1970 \quad$ "Vienna Radium Institute Radiocarbon Dates I". En: Radiocarbon, Vol. 12, No 1, pp. 298 - 318.

1984 "Vienna Radium Institute Radiocarbon Dates XIV". En: Radiocarbon, Vol. 26, N³, pp.441448.

\section{FUNG PINEDA, Rosa}

1988 "The Late Preceramic and Initial Period". En: Peruvian Prehistory. Richard Keatinge editor. pp. 67-96. Cambridge University Press.

1982 "El Temprano Surgimiento en el Perú de los Sistemas Socio-Políticos Complejos: Planteamiento de una Hipótesis de Desarrollo Original". En: Primer Simposio de correlaciones antropológicas andino mesoamericano. J. Marcos y P. Norton Eds. ESPOL. Guayaquil. Ecuador.

GARANGER, José

1992 La Prehistoire dans le monde (Nouvelle édition de La Prehistoire de André Leroi . Gourhan). Obra colectiva dirigida pr José Garanger. Serie Nouvelle Clio L'Histoire et ses problémes. Presses Universitaires de France. Paris.

GRIEDER, Terence, Alberto BUENOM., C. EARLE SMITH y Robert MALINA

1988 "La Galgada, Peru. A preceramic culture in transition". University of Texas Press. Austin.
IZUMI, Seinchi y Toshihiko SONO

1963 Andes 2: Excavations at Kotosh, Perú, 1960. Kadokawa Publishing Co. Tokyo.

KIGOSHI, Kunihiko, Yoshio TOMIKURA y Kunihiko ENDO

1962 "Gakushuin Natural Radiocarbon Measurements I".En: Radiocarbon, Vol. 4, pp. 84-94.

KLEIN, Jeffrey, J.C. LERMAN, P.E. DAMON y E.K.RALPH

1982 "Calibration of Radiocarbon Dates: Tables based on the consensus of the Workshop on Calibrating the Radiocarbon Time Scale". En: Radiocarbon, Vol. 24, N², pp. $103-150$.

KOBAYASHI, Hiromi, Taeko HIROSE, Miyoi SUGINO y Naotune WATANABE

1974 "University of Tokyo Radiocarbon measurements V". En: Radiocarbon. Vol. 16, $\mathrm{N}^{\circ}$ 3, pp. $381-387$.

\section{KRUEGER Harold W. y C. Francis WEEKS}

1966 "Geochron Laboratories INC. Radiocarbon Measurements II". En: Radiocarbon, Vol, 8, pp. $142-160$.

KULP, J. Laurence, Herbert W. FEELY y Lansing E. TRYON

1951 "Lamont Natural Radiocarbon Measurements I". En: Science. Vol. 114, N ${ }^{\circ}$ 2970 , pp. $565-568$.

KULP, J. Laurence, Lansing E. TRYON, Walter R. ECKELMAN y William A. SNELL

1952 "Lamont Natural Radiocarbon Measurements II". En: Science, Vol 116, N ${ }^{\circ}$ 3016, pp. 409 - 414.

LANNING, Edward

1963 "A ceramic sequence for the Piura and Chira coast, north Perú". En: University of California Publications in American Archaeology and Ethnology. Vol. 46, $\mathrm{N}^{\circ} 2$, pp 135 - 284.University of California Press. Eerkeley and Los Angeles.

LILIU, Chao, Kerry M. RILEY y Dennis D. COLEMAN 1986 "Illinois State Geological Survey radiocarbon dates VIII". En: Radiocarbon, Vol. 28, N 1, pp. $78-109$.

LUMBRERAS, Luis Guillermo

1981 La Arqueología como Ciencia Social. Lima. Ediciones PEISA.

1989 Chavín de Huántar en el Nacimiento de la Civilización Andina. Lima. Ediciones INDEA. 
LIBBY, W.F.

1970 "Ruminations on radiocarbon dating". En: Radiocarbon Variations and Absolute Chronology. Nobel Symposium 12. Proceedings of the Twelfth Nobel Symposium held at the Institute of Physics at Uppsala University. Ingrid U. Olsson, Editor. Pp.629640. Estocolmo. Almqvist \& Wiksell y Wiley Interscience Division.

MENZEL, Dorothy

1971 "Estudios arqueológicos en los valles de Ica, Pisco, Chincha y Cañete". En: Arqueología y Sociedad. $\mathrm{N}^{\circ}$ 6. Museo de Arqueología y Etnología de la Universidad Nacional Mayor de San Marcos. Lima.

MENZEL, Dorothy, John ROWE y Lawrence DAWSON 1964 "The Paracas Pottery of Ica A Study in Style and Time". En: University of California Publications in American Archaeology and Ethnology. Vol. 50. Berkeley.

MOOK, W. G. y H. T. WATERBOLK

1985 "Radiocarbon Dating". En : Handbooks for Archaeologists. $\mathrm{N}^{\circ} 3$. European Science Foundation. Strasbourg.

NEUSTUPNY, Evzen

1970 "A New Epoch in Radiocarbon Dating". En : Antiquity. Vol. 45, $\mathrm{N}^{\circ} 173$, pp. $38-45$.

OLIVE, Philippe.

1988 Datation, par le radiocarbone, de materiaux archéologiques (bois, tourbes, ossements). Publication C.R.G. $n^{\circ} 332$. Francia.

\section{OLSON, Edwin A. y W.S. BROECKER}

1959 "Lamont Natural Radiocarbon Measurements V". En: Radiocarbon. Vol. 1, pp. 1-28.

OLSSON, Ingrid U.

1982 "On the Work of the International Committee in Calibration of the Radiocarbon Dating Time Scale" . En : PACT, $\mathrm{N}^{\circ} 7$, part 1, pp. 37 - 43. Actas del "Second Nordic conference on the application of Scientific Methods in Archaeology. Dinamarca, 17 - 19 agosto, 1981. Strasbourg. Conseil d'Europe.

POZORSKI, Shelia

1987 "Theocracy vs. militarism: the significance of the Casma Valley in understanding early state formation". En: The origins and development of the Andean state, J. Haas, S. Pozorski y T. Pozorski, editores. pp.15 - 30. Cambridge University Press.
POZORSKI, Thomas y Shelia POZORSKI

1987 "Chavin, the Early Horizon and the Initial Period". En : The origins and development of tile Andean state, J. Haas, S. Pozorski y T. Pozorski, editores. pp. 36 - 46. Cambridge University Press.

RAVINES, Roger y Juan José ALVAREZ S.

1967 "Fechas radiocarbónicas para el Perú". En: Arqueológicas, $\mathrm{N}^{\circ}$ 11. Lima. Museo Nacional de Arqueología y Antropología.

RENFREW, Colin

[1979] Les origines de l'Europe, la revolution du 1983 radiocarbone. Traducción francesa de "Before Civilization". Flammarion, Paris.

RICHARD, P, B. GUILLET, R. JAEGY y R. COPPENS

1978 "Nancy Natural Radiocarbon Measurements IV". En: Radiocarbon, Vol. 20-1, pp. 62 - 67.

SATO,J., T. SATO, Yasuko OTOMORI y H. SUZUKI

1969 "University of Tokyo Radiocarbon measurements II". En: Radiocarbon, Vol. 11, N'2, pp. $509-517$.

SHIMADA, Izumi, Carlos ELERA y Víctor CHANG J. 1990 "Excavaciones en Hornos de cerámica de la época Formativa en Batan Grande, Costa Norte del Perú". En: Gaceta Arqueológica Andina Vol. 5, №20, pp. 19-43. Lima. INDEA.

STUTVER, Minze y Thomas F. BRAZIUNAS.

1993 "Modeling Atmospheric ${ }^{14} \mathrm{C}$ Influences and ${ }^{14} \mathrm{C}$ Ages of Marine Samples to 10,000 BC". En: Radiocarbon. Vol. 35, N 1.pp.137- 189. Department of Geosciences The University of Arizona. Tucson, Arizona.

STUIVER, Minze, Gordon.W. PEARSON y Thomas F. BRAZIUNAS

1986 "Radiocarbon age calibration of marines samples back to $9000 \mathrm{cal}$ yr BP". En: Radiocarbon, Vol. 28, $\mathrm{N}^{\circ} 2 \mathrm{~B}$, pp. 980 1021

STUIVER, Minze y Paula REIMER.

1993 "Extended ${ }^{14} \mathrm{C}$ Data Base and Revised Calib $3.0{ }^{14} \mathrm{C}$ Age Calibration Program". En : Radiocarbon. Vol. 35, $\mathrm{N}^{\circ} 1$. pp. 215 - 230. Department of Geosciences The University of Arizona. Tucson, Arizona.

1994 CALIB ver. 3.0.3.A. Manuel sur l'utilisation du programme de calibration et commentaires utiles. Seattle. Quaternary Research Center AK - 60. Traducción en francés de P. Fortin. 
TRAUTMAN, Milton A. y Eric H. WILLIS

1966 "Isotopes, INC. Radiocarbon Measurements V". En: Radiocarbon. Vol. 8, pp. 161 - 203.

VALASTRO, S., E. MOTT DAVIS y A. VARELA

1978 "University of Texas at Austin Radiocarbon Dates VII". En: Radiocarbon. Vol. 20, N 2 , pp. 245- 273.

VALASTRO, S., E. MOTT DAVIS,A. VARELAy S.V. LISK

1986 "University of Texas at Austin Radiocarbon Dates XV". En: Radiocarbon. Vol. 28, ㅇ3, pp. 1173-1199.

VALLADAS, Helene. Maurice ARNOLD y Pierre MAURICE

1989 "La Datation Carbone 14 en Spectomètrie de Masse par Accelerateur". En : Le Temps de la Préhistoire, pp.211 - 212. Dijon. SPF.

VÁSQUEZ, José

1984 "El carbono 14 y las culturas del antiguo Perú". En: Revista peruana de Física III-1. pp. 119-134. Sociedad Peruana de Física, Lima.
VOGEL, J. C., y J. C. LERMAN

1969 "Groningen Radiocarbon Dates VIII". En: Radiocarbon. Vol. 11, №2. pp. 351 - 390.

VOGEL, J. C., Annemarie FULS, Ebbie VISSER y Bernd BECKER

1993 "Pretoria Calibration Curve for Short - Lived Samples, 1930 - 3350 BC". En: Radiocarbon. Vol. 35, $\mathrm{N}^{\circ}$ 1. pp. 73 - 85. Department of Geosciences The University of Arizona. Tucson, Arizona.

YAMASAKI, Fumio, Tatsuji HAMADA y Chikako FUITYAMA

1966 "Riken Natural Radiocarbon Measurements II". En: Radiocarbon. Vol. 8, pp. 324 - 339.

ZIOLKOWSKI, Mariusz S., Mieczyslaw F. PAZDUR, Andrzej KRZANOWSKI y Adam MICHCZYNSKI

1994 Andes, Radiocarbon Database for Bolivia, Ecuador and Peru. Varsovia y Glivice. Publicación conjunta del Andean Archaeological Mission of the Institute of Archaeology, Warsaw University \& Gliwice Radiocarbon Laboratory of the Instituts of Physics, Silesian Technical University. 


\title{
FIGURINAS FUNERARIAS DE LA CULTURA LIMA
}

\author{
Augusto Amador Parodi.
}

\section{Sumilla:}

Este articulo intenta entender la función y significado de un grupo de figurinas antropomorfas de cerámica, partiendo de sus caracteristicas fisicas y de su ubicación al interior de contextos funerarios, provienentes de un cementerio excavado en el sitio Cerro Culebra, Costa Central, que corresponde a la cultura Lima del Periodo Intermedio Temprano. Casi no se conocen ejemplos asignables a dicha cultura. La característica principal de estas representaciones es la repetición de un esquema basado en facciones humanas elementales, prescindiéndose de detalles. Esto marca una diferencia con las tendencias observadas en figurinas antropomorfas de otras épocas. A partir de nuestro análisis, se infiere su carácter funerario relacionado al trance, de la muerte, y sus criterios de identidad grupal.

\section{Abstract:}

This paper tries to understand the function and significance of a group of anthropomorfic figurines, since their physical features and their location inside funerary contexts. The samples belongs to Cerro Culebra, an archaeological site of the central coast. With almost no other examples, their principal characteristic is the repetition of elemental human features, with almost no details. This is a important difference with human figurines of other periods. With our research, we propose a funerary character related with the moment of death and group identity judgements.

El termino "figurina" se refiere a representaciones humanas que no son vasijas (huacos) ni parte de ellas...

Alexandra Morgan, 1991: 156

\section{INTRODUCCIÓN}

Las figurinas, en sus diferentes tamaños, formas, materiales y estilos; han sido un elemento presente a través de casi todo el desarrollo cultural de los Andes. Las más comunes, variadas y complejas son, sin duda, las fabricadas con arcilla, sea ésta cocida o no. Se las ha encontrado en los más diversos contextos. desde rellenos arquitectónicos, basurales, arquitectura monumental, hasta, por supuesto, contextos funerarios.

Pocos estudios se han realizado sobre el tema: enfocados en tipologías o tratando de entenderlas en un contexto particular. La mayoría de las veces únicamente se las menciona como parte del análisis del material cerámico de un sitio específico.

\section{FIGURINAS LIMA}

Los únicos datos publicados sobre figurinas atribuibles a la cultura Lima, son presentados por Jijón y Caamaño. Corresponden a dos piezas procedentes de sus excavaciones en las huacas de Maranga (Jijon y Caamaño, 1949: 473). Tal vez, por esta escasez de datos, Alexandra Morgan piensa que, dentro de esta cultura, prácticamente no se confeccionaron; situación bastante interesante puesto que, justamente en el Intermedio Temprano, culturas como Nasca y Moche alcanzaron gran desarrollo en tal rubro (Morgan, 1991: 156).

\section{a) Cerro Culebra}

Las excavaciones de rescate llevadas a cabo en el cementerio de la Quebrada II del sitio Cerro Culebra (Fig. 1), dur.unte los primeros meses de 1993, sacaron a la luz una muestra de seis figurinas correspondientes a los entierros de tres diferentes individuos, sepultados según el típico patrón funerario Lima (Falcón, 1993). Estas figurinas sirvieron como punto de partida para la investigación, tema del presente artículo.

De las seis figurinas encontradas, cuatro pertenecían al entierro 2, una al entierro 12 , y la otra al entierro 40. A continuación, se describen las figurinas del entierro 2 :

$A$ y $B$ : Dos figurinas de cuerpo semiesférico, cuyos únicos rasgos representados son: la nariz modelada, el mentón y la boca incisa. No presentan ojos, brazos, ni piernas. Sus narices comienzan en el extremo superior de la cabeza, que tiende a ser recto, contrastando con el tratamiento general del rostro, que es redondeado, con énfasis en el mentón. Son macizas y el acabado de superficie es burdo (Figs. 2 y 3 ).

C: Una figurina pequeña cuyo cuerpo tiene

Augusto Amador Parodi. Pontificia Universidad Católica del Perú. Lima. E-mail: a9120139@pucp.edu.pe 


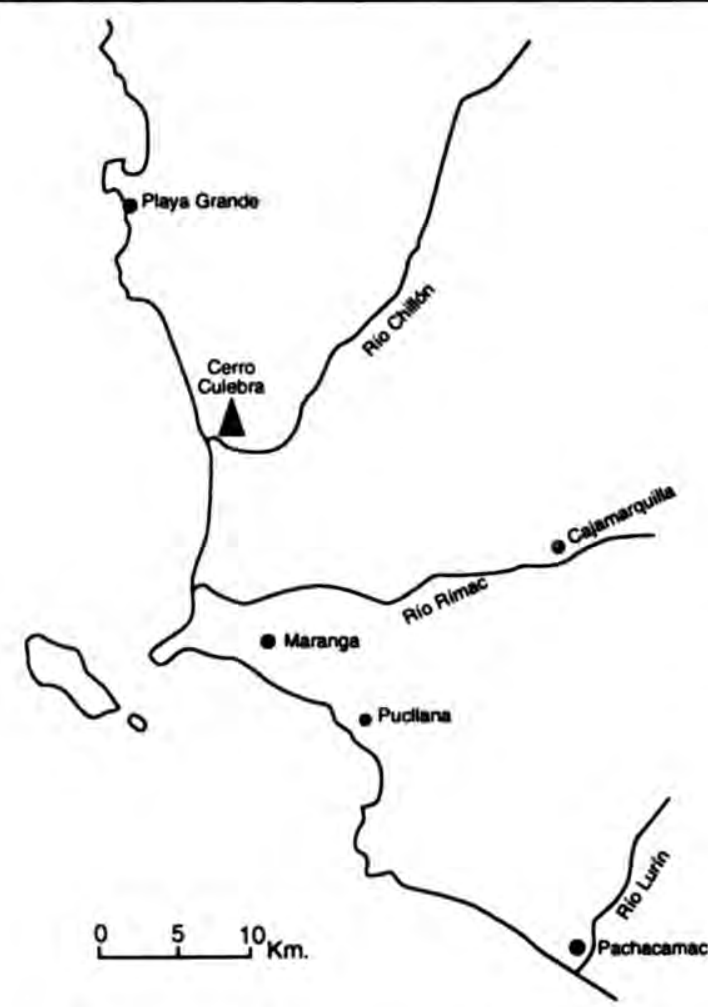

Fig. I Ubicación del sitio Cerro Culebra y otros sitios de la cultura Lima.

forma de cono invertido, al cual se añade una pequeña cabeza en la que destacan, como únicos rasgos, una nariz modelada y un mentón bastante más fino que los de las anteriores. En la parte inferior-posterior, una incisión tosca sugiere la división entre las piernas (Fig. 4).

D: Una figurina un tanto más convencional. La cabeza coincide con las primeras en destacar el mentón y tener un extremo superior recto. La nariz es modelada. Se representan piernas cortas, aunque no brazos. Debajo del espacio en el que idealmente deberían estar los brazos, un estrecho agujero corre de costado a costado. evidenciando cierto vacío interior. El cuerpo es más antropomorfo que los anteriormente vistos (Fig. 5).

En cuanto al Entierro 12:

E: Una figurina que repite la característica del espacio vacío en su interior y los pequeños agujeros laterales. La nariz es igualmente prominente, y presenta piernas cortas, menos acabadas que la anterior. La tendencia rectilínea del extremo de la cabeza es interrumpida por una elevación a cada lado que, a primera vista, podrían dar la impresión de ser orejas animales. En este caso, no está marcado el mentón como un volumen que sobresale, aunque el contorno redondeado del rostro es igualmente perceptible, e inmediatamente debajo del rostro, el cuerpo se agloba hasta antes de las piernas (Fig. 6).

En el Entierro 40 se encontró:

F: Una figurina formalmente parecida a las últimas, pero de un acabado diferente. La nariz modelada empieza en la parte superior de la cabeza, que en dicho extremo recto pronuncia sus ángulos, resaltando aún más la curvatura del contorno del rostro. También tiene los agujeros que anuncian que la pieza es parcialmente hueca, ubicados debajo de los hombros, ante la ausencia de brazos. La principal característica de esta pieza es que presenta decoración pintada (motivo interlocking) sobre el cuerpo, tanto al frente como en la espalda, exceptuando las piernas y la cabeza. También, con pintura, están señalados la boca y, por primera vez, los ojos (Fig. 7).

Poco detalle en las facciones, así como un acabado burdo, son el común denominador en prácticamente todas las figurinas vistas; un ligero alisado complementa a una cocción limitada; no hay engobes y sólo una está pintada. La figurina $\mathrm{F}$ es, en ese sentido, la mejor lograda, presenta bruñido, $y$ pareciera fabricada con mayor esmero (quizá no por falta de conocimiento de la técnica). Particularmente, las tres últimas dejan la impresión de una cierta mirada hacia arriba que las lleva a empinar la nariz y pronunciar el mentón.

\section{b) Maranga}

El referente más cercano para las piezas vistas, lo conforman las dos figurinas de las huacas de Maranga, excavadas por Jacinto Jijón y Caamaño (1949: fig. 217 y 218):

-La primera; cuadrangular, hueca, sin engobe, ligeramente alisada y con perforación de costado a costado. El único rasgo observable es la nariz. Corresponde al entierro CVII (Fig. 8).

-La segunda; en la que se diferencia la cabeza del cuerpo, también tiene perforación y la nariz como único rasgo. Corresponde al entierro CLXI (Fig. 9).

En ambos casos, parece ser que el arco que se forma en el extremo inferior del cuerpo está insinuando piernas. Además, la primera de estas dos, muestra los costados del extremo superior de la cabeza más prominentes que el centro.

\section{CARÁCTER FUNERARIOYESQUEMATISMODE LAS FIGURINAS}

Las ocho figurinas que estamos reconociendo como Lima, provienen en su totalidad de contextos funerarios. En las excavaciones que desde Uhle se han realizado, incluyendo las más recientes, no existe evidencia de hallazgos de figurinas, ni parecidas, $\mathrm{ni}$ diferentes a éstas, ni identificables con esta cultura en ningún otro tipo de contexto; nada más allá de las que aquí se presentan. Así, hasta el día de hoy, el universo absoluto de figurinas Lima proviene de contextos funerarios.

Como ya hemos dicho antes, la manufactura de estos objetos es bastante simple, los materiales no son 


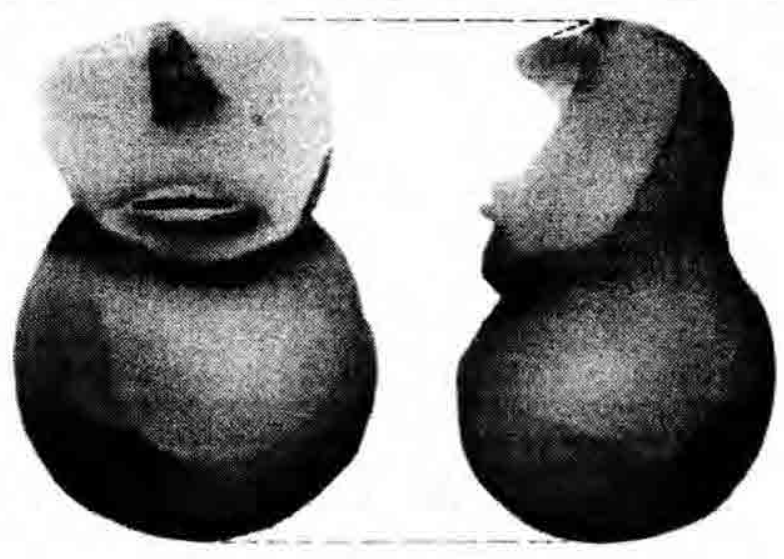

Fig. 2 Figurina A de Cerro Culebra

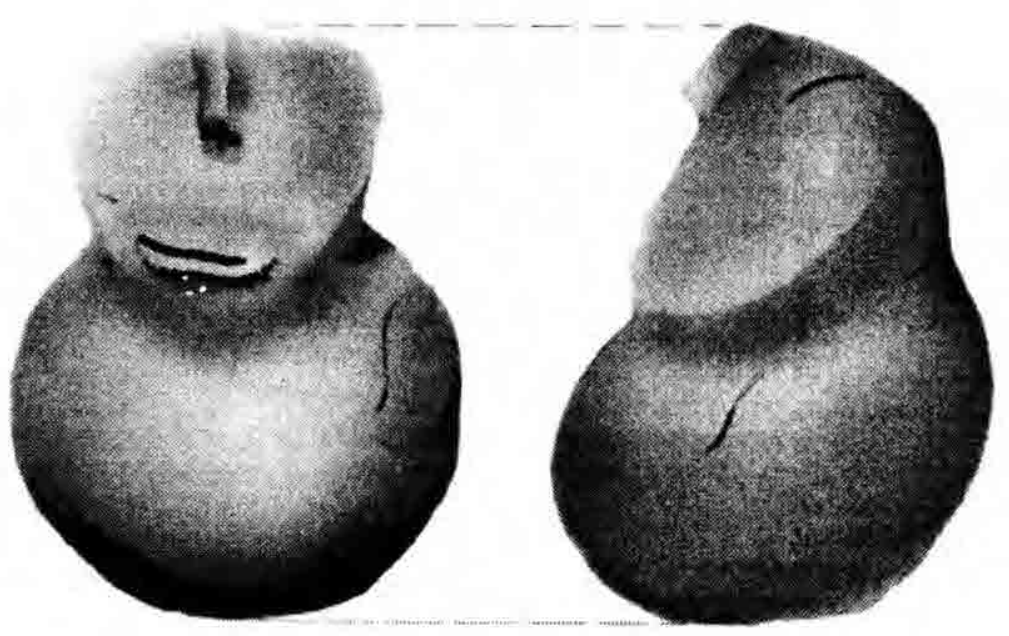

Fig. 3 Figurina B de Cerro Culebra
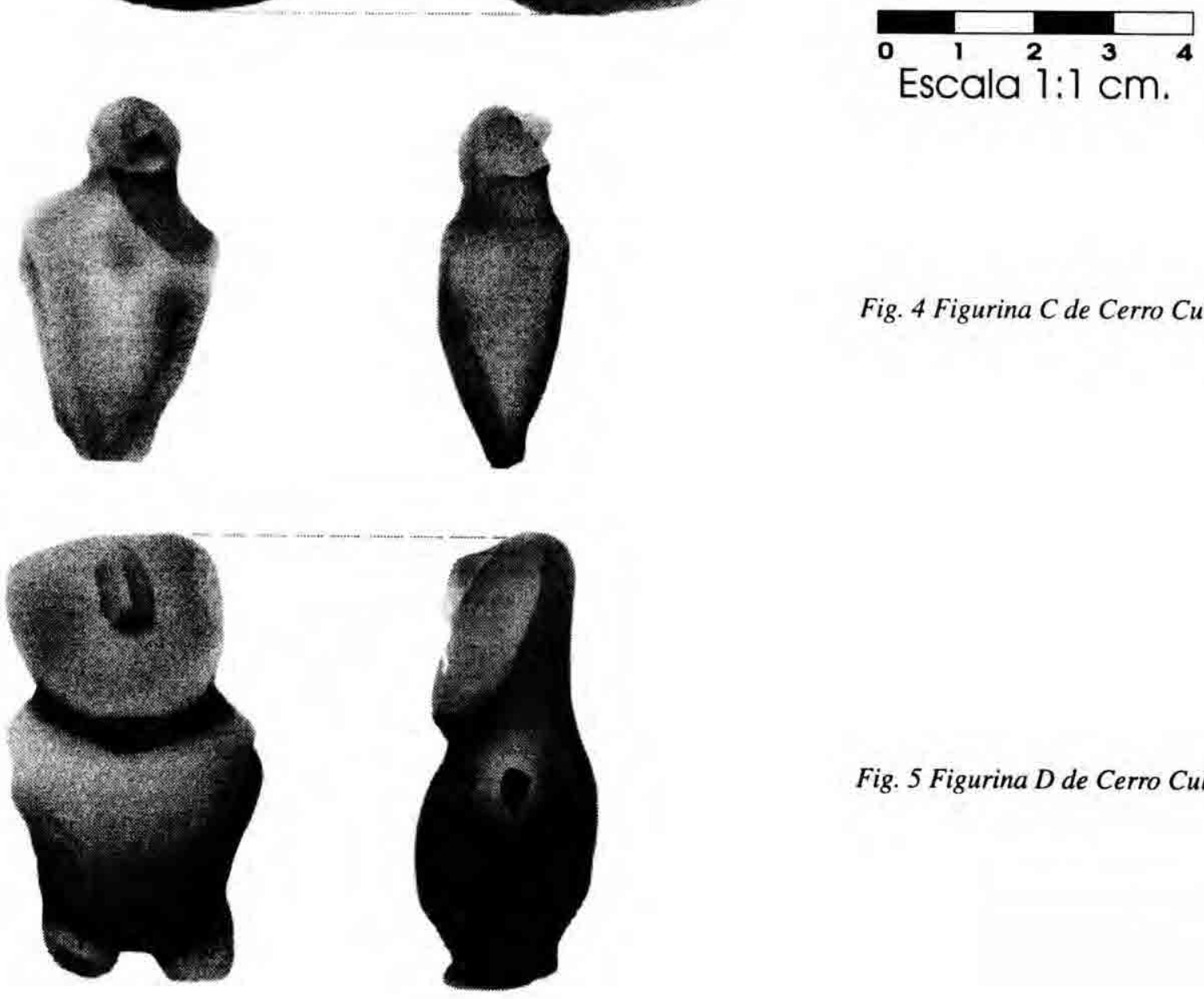

Fig. 4 Figurina C de Cerro Culebra

Fig. 5 Figurina D de Cerro Culebra 
muy resistentes ni están bien cocidos; todo ésto los convierte en sumamente vulnerables, imaginados en un uso cotidiano. Muchas veces se ha querido identificar en las figurinas a juguetes que, llegado el caso, eran depositados en las tumbas con sus usuarios en vida. Sea como fuere, en nuestra muestra no se observan huellas de un uso anterior a su deposición; por el contrario, las piezas aparecen enteras y bien conservadas.

Por todo ello, definiremos a este grupo como "de carácter funerario"; es decir, objetos hechos y destinados exclusivamente para el rito funerario. Es claro que no existe en estos objetos una intención de representar fielmente la figura humana, ni menos aún de retratar a algún o algunos personajes determinados; por el contrario, se trata de un esquematismo basado en rasgos humanos esenciales, como son la nariz y el mentón, además de la proporción entre cuerpo y cabeza. Eventualmente, se adicionan o se esbozan boca, piernas $y$, en un caso, ojos. Se suele representar un rostro más o menos estereotipado $y$, aunque existan diferencias de una a otra figurina, todas parecen estar bajo una misma idea general.

Es muy interesante observar cómo esos dos elementos fundamentales en el esquema representativo de las figurinas Lima (nariz-mentón), juegan también un importante papel en otros objetos de naturaleza funeraria, tales como las máscaras o falsas cabezas que se popularizan en los fardos durante el Horizonte Medio (Ravines, 1981). No suele faltar la nariz grande de madera o hecha de tela. Las máscaras de madera suelen dar también importancia al mentón redondeado (Wiener, 1880: pp. 71, 691).

Otros objetos donde se destacan esos rasgos son los sarcófagos de arcilla del Utcubamba. Precisamente, Kauffmann Doig sugiere una relación entre éstos y las mencionadas máscaras (Kauffmann Doig, 1989: lám. IV, pp. 24-25). Los sarcófagos reproducen la misma idea de representación estereotipada, con gran nariz y mentón redondeado que sobresale y se distingue del resto del cuerpo.

Finalmente, están las horquetas de madera con representaciones de rostros tallados, culturalmente consideradas Ica-Chincha (Kauffmann Doig y Chumpitaz, 1993: fig. 10b). Éstas conservan muchas veces pigmento rojo sobre el rostro, de reconocido significado funerario. Wiener muestra una en un dibujo, emplazada en medio de una cámara funeraria, detrás del fardo (Wiener, 1880: pp. 47). Tales horquetas, si bien suelen incluir más rasgos, son también representaciones esquemáticas en las que se destacan la nariz y el mentón.

Todos los objetos mencionados corresponden a períodos cronológicos posteriores al Intermedio Temprano.

\section{DISCUSIÓN}

Un aspecto que llama la atención en las figurinas Lima, es la no inclusión de atributos sexuales, tan comunes en figurinas de períodos anteriores, pero sobre todo en las posteriores. La cuestión del género parece no tener relevancia en el significado fundamental del objeto.

La perforación que atraviesa a varias de ellas, debió favorecer la cocción pero en ningún caso existe evidencia de que haya servido para pasar una cuerda, como lo sugiere Jijón y Caamaño (1949: 473). La presencia de una cuerda nos hablaría de un medio para portar con facilidad las piezas, ya sea en vida (posibilidad negada), o en la muerte, muy probablemente sujetadas al cuerpo. Se conoce con precisión la ubicación original de cinco figurinas, y ella no concuerda con tales ideas; ya que se encontraban fuera del fardo y cercanas a la cabeza del difunto.

En un intento por explicar el significado de las figurinas, Jijón y Caamaño postula que éstas pudieron ser sustitutos de sacrificios humanos, depositadas en los entierros como una forma de economía funeraria, es decir, que al no poder disponer de una vida humana para el sacrificio, se recurría a este artificio. Sin embargo, no existe evidencia en ese sentido; por el contrario, el entierro 40 de Cerro Culebra, 0 además de una figurina, presentaba un aparente sacrificio humano; y los entierros con figurina podrían estar mostrando algunos objetos, como cuentas de Spondyllus, cuyo valor relativo estaría contradiciendo una supuesta pobreza (Falcón, 1993; Falcón y Amador, 1997).

Los tres individuos cuyas tumbas contenían figurinas, cn Cerro Culebra, eran niños y, de los de Maranga, por lo menos uno también. La edad del último de los de Maranga no queda clara debido a lo enmarañado de la descripción que hace el autor. Pero el dato disponible ya es bastante ilustrativo: ¿necesitarían particularmente los más pequeños de este elemento?

Más allá de la cuestión de la edad, la ocurrencia de sólo tres individuos con figurina, entre más de cuarenta excavados para Cerro Culebra, y de sólo dos entre varias decenas de Maranga; nos indica que éstas no eran colocadas de manera libre; lo que se ve reforzado por el pequeño número de piezas recuperadas. Algún principio cultural debió regular este patrón funerario de colocar o no una figurina.

Habíamos mencionado la forma que tiene el extremo superior de la cabeza, sobre todo en tres de las figurinas: E, F y la primera de Jijón y Caamaño (1949: fig. 217). Dicha parte parece expandirse hacia los lados, generando una protuberancia en cada uno. Dado que las representaciones son tan simples y no se esfuerzan 

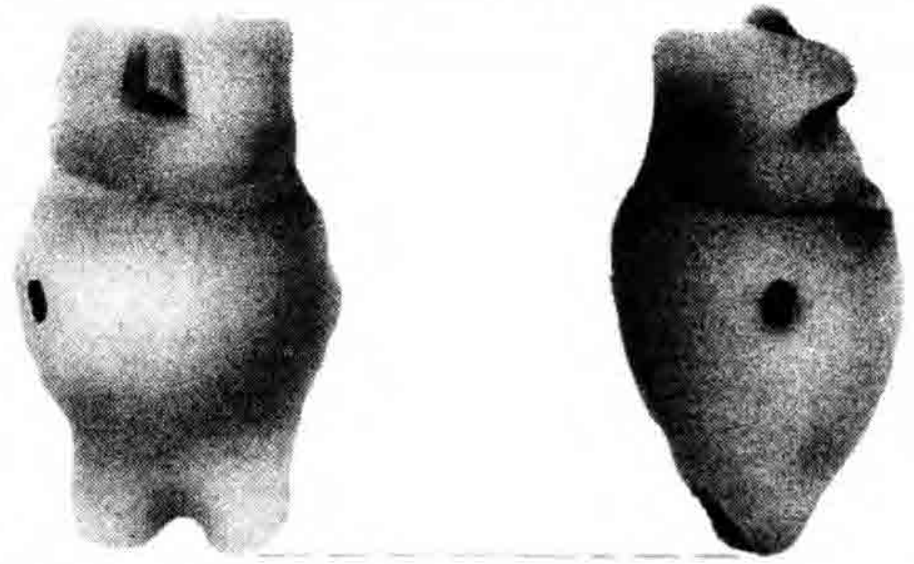

Fig. 6 Figurina E de Cerro Culebra
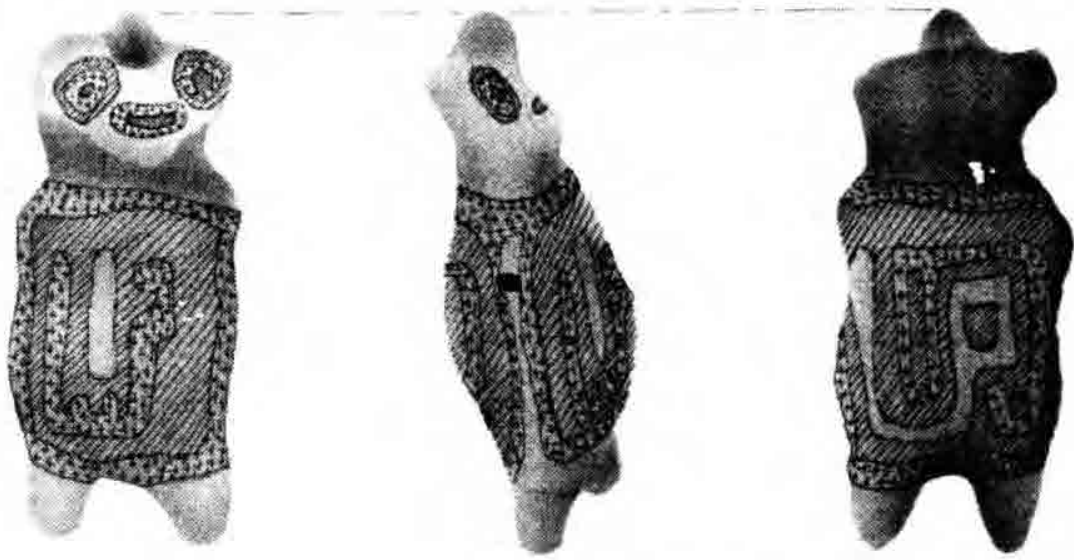

Fig. 7 Figurina $F$ de Cerro Culebra

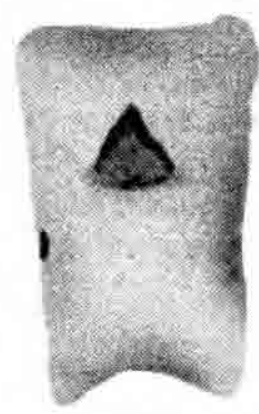

Fig. 8

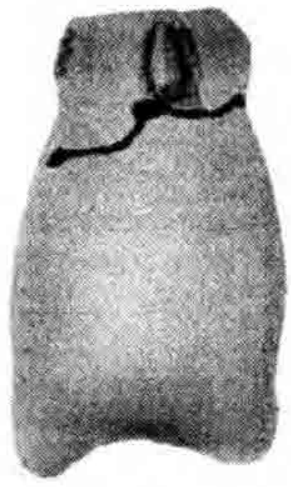

Fig. 9
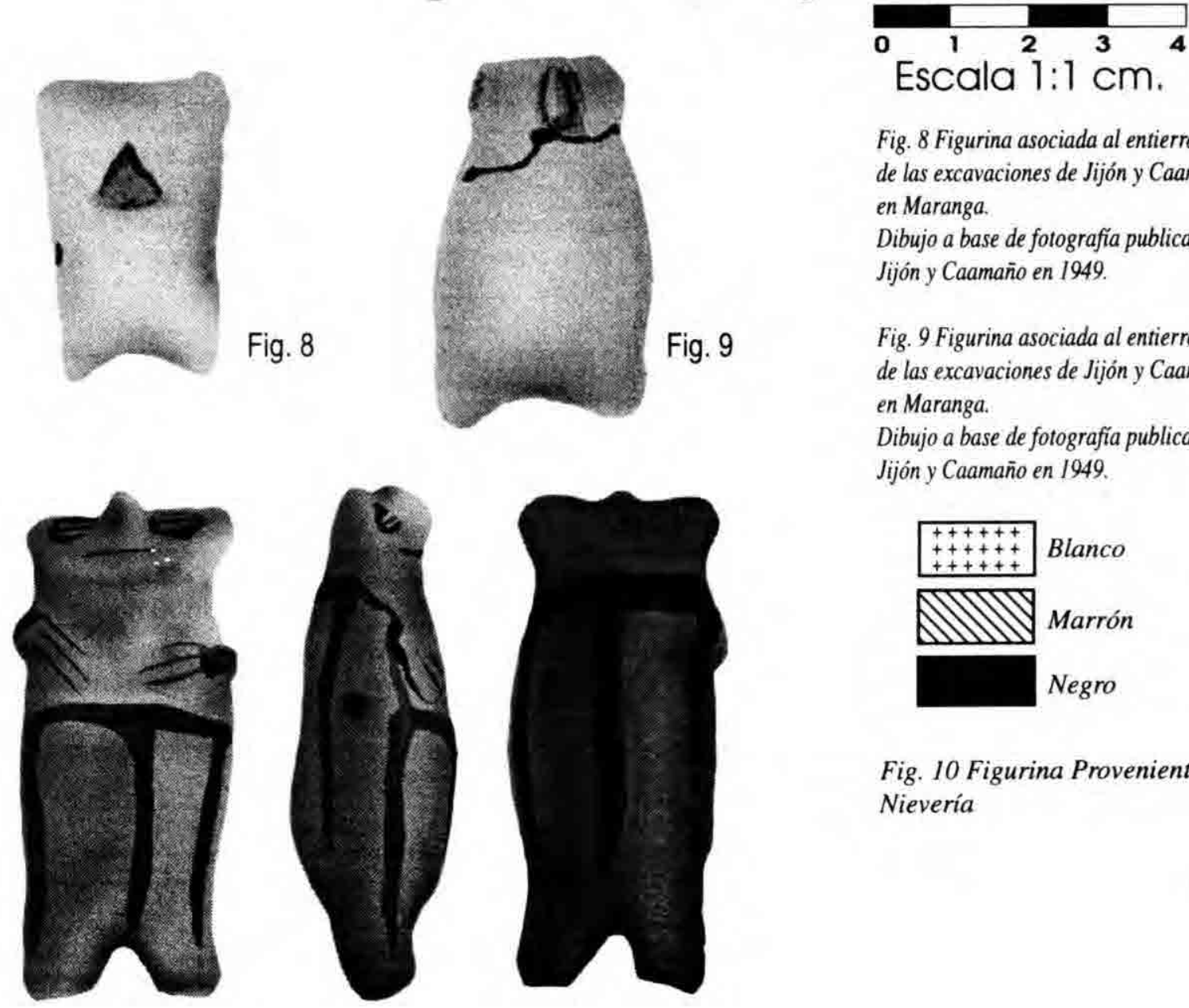

Fig. 8 Figurina asociada al entierro CVII, de las excavaciones de Jijón y Caamaño en Maranga.

Dibujo a base de fotografia publicada por Jijón y Caamaño en 1949.

Fig. 9 Figurina asociada al entierro CLXI de las excavaciones de Jijón y Caamaño en Maranga.

Dibujo a base de fotografia publicada por Jijón y Caamaño en 1949.

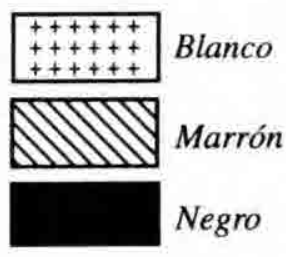

Fig. 10 Figurina Proveniente de Nievería 
ni siquiera en mostrar partes del cuerpo, sería demasiado pensar que tales protuberancias están mostrando alguna clase de tocado o gorro, ya que en ningún caso se representa.

Siendo que lo que se expresa es más bien un arquetipo, una forma básica humana, con la cual deben haberse identificado los miembros del grupo, no sería descabellado pensar que se trate de una deformación craneana de tipo Huaura, variante bilobada (Weiss, 1962) que se encuentra presente en la colección ósea de Cerro Culebra, y que debió parecerles familiar.

\section{a) Figurinas cronológicamente cercanas}

Existen algunos especímenes de los valles próximos, cronológicamente cercanos, que comparten algunas características de las que hemos definido para la cultura Lima.

Palacios (1988: fig. 23) presenta una figurina que ubica en su fase Huayco Medio de Huachipa y que sería inmediatamente anterior a Lima, como allí mismo se afirma. Esta figurina tiene piernas muy similares a las de Lima de nuestra muestra y también destaca su nariz, pero cuenta con ojos y boca incisos, así como brazos extendidos al frente. El efecto visual del acabado es también bastante parecido. No tiene un contexto definido.

En Tablada de Lurín también aparecen figurinas en entierros, como la presentada por Ramos de Cox en el Boletín del Seminario de Arqueología del Instituto Riva Agüero (Ramos de Cox, 1971: láms. I y IIIb) que correspondería probablemente a fines del Formativo. Ésta tiene importantes semejanzas con nuestra muestra Lima, como: la actitud de mirar hacia arriba con el mentón pronunciado, la nariz grande, las piernas, la forma del cuerpo, los agujeros laterales y el vacío, etc. La principal diferencia radica en la presencia de unos brazos muy cortos, extendidos hacia los lados, y los rasgos faciales incisos: ojos, fosas nasales y boca.

Cosa parecida ocurre con el período inmediato posterior, Nievería, de los inicios del Horizonte Medio.

Un interesantísimo hallazgo realizamos en los depósitos de cerámica del Museo Nacional de Arqueología, Antropología e Historia del Perú; allí encontramos una figurina proveniente del cementerio al sur del Río Seco, sitio de Nievería (Fig. 10). Esta figurina tiene un sorprendente parecido con la denominada $\mathrm{F}$ de Cerro Culebra. Formalmente son muy parecidas, siendo la de Nievería un poco más grande y con brazos que dobla sobre su pecho. La de Nievería tiene un color anaranjado y la de Cerro Culebra rojizo. La decoración es diferente. Por otro lado, la figurina encontrada en el Museo también posee orificios laterales, similitud que, al lado de las diferencias, revela la existencia de continuidades en las costumbres que hemos visto en la época Lima.

Para el sitio Potrero Tenorio, en Cantogrande, Palacios y Guerrero muestran una figurina fragmentada que nos recuerda vivamente a las figurinas A y B de nuestra muestra; aunque ésta tiene boca y brazos sobre el pecho (Palacios y Guerrero, 1992: fig 6).

Las recientes excavaciones en el sitio de Cajamarquilla, valle del Rímac, a cargo de Juan Mogrovejo, trajeron a la luz el contexto funerario número ocho de la época Nievería que contenía una figurina sumamente burda (J. Mogrovejo, comunicación personal, abril de 1997). Ésta muestra solamente la nariz y, al parecer, brazos sobre el pecho, como rasgos de su naturaleza antropomorfa. Está hecha de arcilla no cocida.

\section{CONCLUSIONES}

La. figurinas pertenecientes a la cultura Lima que forman nuestra muestra, pueden ser consideradas de carácter funerario, partiendo de sus contextos de origen $y$ ante la evidencia de haber sido fabricadas exclusivamente para el entierro.

La muestra constituye un grupo consistente, con ciertas variaciones formales, pero con un concepto compartido. Tales variaciones podrían deberse a preferencias individuales en la manufactura, al no ser siempre un especialista quien las hace sino que, al parecer, más bien se fabricaron ante la eventualidad funeraria y por manos de los involucrados directos (probablemente deudos), quienes no necesariamente eran buenos conocedores de la técnica. A la vez, ello supone cierta flexibilidad en el patrón.

El punto común en el cual se tocan todas estas figurinas, es un esquema de representación de la figura humana simplificada al máximo que, posiblemente, incluya un elemento de identidad cuya naturaleza habría que esclarecer. Uno de ellos podría ser la deformación craneana.

Esto último nos mueve a reflexionar sobre la función ritual de estas figurinas dentro de los contextos funerarios, más aún si consideramos que esos contextos corresponden a niños. Tal vez se consideraba necesario acompañar a ciertos niños con estas representaciones de la identidad de su grupo social o familiar, para acercarlos a los ancestros comunes.

Parece ser que estas figurinas, como expresión de un ideal, de un arquetipo, comparten cierto nivel de significación con las ya mencionadas máscaras funerarias y otros elementos funerarios similares. Es interesante 
ver que, en tiempos más tardíos, cuando tales máscaras, horquetas y sarcófagos se vuelven populares, las figurinas de las distintas culturas se hacen más diversas y complejas, llegando a constituir posibles personajes y perdiendo esa característica sumaria propia de las figurinas Lima.

De la observación de las figurinas inmediatamente anteriores e inmediatamente posteriores, se puede atisbar la existencia de una tradición de figurinas funerarias, que se inicia en el Formativo, abarca la época Lima y se proyecta hacia Nievería, donde la fabricación de figurinas en forma sumaria y poco cuidada, tiene su correlato en el hallazgo reciente de Cajamarquilla.

\section{Agradecimientos:}

Este artículo no hubiera sido posible sin la generosa colaboración de los siguientes arqueólogos: Víctor Falcón, Martín MacKay, Rafael Santa Cruz, Patricia Arana y Juan Mogrovejo. Agradezco también a Lucy Linares, del Departamento de Cerámica, y a Fanny Montesinos, de Registro y Catalogación del MNAAHP por el apoyo brindado.

\section{BIBLIOGRAFÍA}

FALCÓN HUAYTA, Víctor

1993 Informe Final.Proyecto de Rescate Arqueológico Cerro Culebra. MNAAHP. Lima.

FALCÓN, Víctor y Augusto AMADOR

1997 "Un Entierro de Patrón Funerario Lima en Cerro Culebra." En: Mauq'a Llaqta $N^{\circ} 1: 51$ 68. Lima.

JIJÓN y CAAMAÑO, Jacinto

1949 Maranga. Contribución al conocimiento de los aborígenes del valle del Rimac. Perú. La Prensa Católica. Quito.

KAUFFMANN DOIG, Federico

1989 "Expedición Antisuyo 87". En: Arqueológicas 20. MNAA. Lima.

KAUFFMANN, Federico y Evaristo CHUMPITAZ

1993 "Exploración del Templo Pintado del Ingenio, Nasca (Perú)". En: Baessler-Archiv, Neue Folge, Band XII. pp. 39-72. Berlín.

MENZEL, Dorothy

1967 "Late Ica figurines in the Uhle collection".
En: Ñawpa Pacha 5: 15-39. Berkeley, California.

MORGAN, Alexandra

1991 "Las figurillas humanas de cerámica de la cultura Chancay". En: Estudios Sobre la cultura Chancay. Perú. A. Krzanowski, Editor. pp. 156-187. Polonia.

MUELLE, Jorge C.

1935 "Restos hallados en una tumba en Nievería". En: Revista del Museo Nacional. T. IV, No 1 : 135-152. Lima.

PALACIOS, Jonathan

1988 "La secuencia de la cerámica temprana del valle de Lima en Huachipa". En: Gaceta Arqueológica Andina 16: 13-24. Lima.

PALACIOS, Jonathan y Carlos D. GUERRERO

1992 "Potrero Tenorio: Un enterramiento ritual de ofrendas del estilo Nievería en el valle del Rímac". En: Pachacamac. N 1: 75-100. Lima.

\section{RAMOS DI COX, Josefina}

1971 "Figurines de Lima: Posibles arquetipos ocupacionales". En:Arqueología PUC. No 11 : 103-108. Lima.

\section{RAVINES, Rogger}

1981 "Prácticas funerarias en Ancón (Segunda parte)". En: Revista del Museo Nacional XLV: 89-166. Lima.

ROSAS LA NOIRE, Hermilio

1970 La secuencia cultural del período formativo en Ancón. Tesis para obtener el grado de Bachiller en Arqueología. UNMSM. Lima.

\section{STOTHERT, Karen y Rogger RAVINES}

197 "Investigaciones arqueológicas en Villa El Salvador". En: Revista del Museo Nacional. XIIII: 157-226. Lima

WEISS, Pedro

1962 "Tipología de las deformaciones cefálicas de los antiguos peruanos según la osteología cultural". En: Revista del Museo Nacional. XXXI:15-42. Lima.

WIENER, Charles

[1880] Perú y Bolivia.

1993 UNMSM. Lima 